\title{
STABILIZATION ARISING FROM PGEM: A REVIEW AND FURTHER DEVELOPMENTS
}

\author{
RODOLFO ARAYA ${ }^{1}$, GABRIEL R. BARRENECHEA ${ }^{2}$, LEOPOLDO P. FRANCA ${ }^{3}$, \\ AND FRÉDÉRIC VALENTIN ${ }^{4}$
}

\begin{abstract}
The aim of this paper is twofold. First, we review the recent Petrov-Galerkin enriched method (PGEM) to stabilize numerical solutions of BVP's in primal and mixed forms. Then, we extend such enrichment technique to a mixed singularly perturbed problem, namely, the generalized Stokes problem, and focus on a stabilized finite element method arising in a natural way after performing static condensation. The resulting stabilized method is shown to lead to optimal convergences, and afterward, it is numerically validated.
\end{abstract}

\section{INTRODUCTION}

The Stokes problem emanates from modeling creeping flows and incompressible elasticity. The problem fits into the abstract mixed method formulation [10,12]. Mixed methods have various applications, among them modeling deformation of beams, arches, plates and shells. The approximation of these problems using standard finite element polynomials faces the challenge of satisfying stability conditions known as inf-sup conditions [10]. These stability conditions restrict which pairs of approximation (primal and dual variables) are allowed. Convenient pairs, such as equal-order interpolations, are in general prohibited.

Stabilized methods address the limitations of mixed methods [29, 28]. Introduced for advective-diffusive problems [16, 20], stabilized methods are built to enhance stability without affecting consistency. This is accomplished by adding terms based on residuals of the equations involving the trial functions while the test functions have different forms varying from least-squares to adjoint operators. For the Stokes problem these methods have been

Date: October 6, 2007.

Key words and phrases. Stokes operator, reactive flow, multiscale function, Petrov-Galerkin method, stabilization.

${ }^{1}$ This author is partially supported by FONDECYT Project No. 1070698.

${ }^{2}$ This author is partially supported by CONICYT-Chile through FONDECYT Project No. 1061032 and FONDAP Program on Applied Mathematics.

${ }^{3}$ This author is partially supported by NSF Grant No. 0610039.

${ }^{4}$ This author is supported by CNPq grant No. 304051/2006-3 and FAPERJ.. 
proved convergent for almost all pairs of interpolation [29, 26]. The drawback of stabilized methods is the choice of stability constants associated with the additional terms. In many applications the value of these constants affects the numerical results.

To shed some light on how to produce the stability constant for the Stokes problem, in [31] a relationship between the enrichment of a piecewise linear velocity field with a bubble function (MINI element [4]) and the stabilized method from [29] was first pointed out. The MINI element produces this stabilized method with a stability constant which is a function of the bubble shape and value. This gives us a recipe for getting the stability constant, namely, we pick a form of the bubble function and this gives us a specific value of the stability constant. This relationship has been extended to the generalized Stokes problem in [8] (see also [6] where this relationship was first highlighted) and to the advective-diffusive problem in $[11,5]$.

The relationship discovery left an open problem, namely how to choose optimal bubbles to produce the most accurate stabilized approximation. This question has been addressed introducing the residual-free-bubbles concept $[15,25,14,13]$. The idea is to construct the bubbles by approximating a local problem dictated by the equations of the global problem. The bubbles solve a PDE problem governed by the residual of the piecewise polynomial component of the solution. The local problem is subject to a zero boundary condition (except for some problems defined in $L^{2}$ ), and this yields good solutions in some applications. However, the zero boundary condition limits the capability of the approximation in some cases. For example in the reactive dominated diffusive model (with, or without convection), the residual-free bubbles method oscillates near a boundary layer. Several solutions have been proposed in order to avoid this type of oscillations, including the use of adaptive meshes or specially refined meshes, such as Shishkin meshes (see [30, 34], and references therein, and [33] for a survey), but the detailed review of these techniques lies beyond the scope of this work. Instead, in this work we focus on so-called Petrov-Galerkin Enriched Method (PGEM) $[22,21]$, which is discussed in the next section.

The remainder of the paper is as follows: a review of PGEM is given in Section 2, in Section 3 PGEM is extended to the generalized Stokes problem, including a stabilized formulation derived from it, for which we perform an a priori error analysis, and in Section 4 we present some numerical results confirming the theoretical results.

1.1. Notations. Let $\Omega$ be an open bounded domain in $\mathbb{R}^{2}$ with polygonal boundary. As usual, $(\cdot, \cdot)_{D}$ stands for the inner product in $L^{2}(D)$ (or in $L^{2}(D)^{2}$, when necessary), and 
we denote by $\|\cdot\|_{s, D}\left(|\cdot|_{s, D}\right)$ the norm (seminorm) in $H^{s}(D)$ (or $H^{s}(D)^{2}$, if necessary). By $\left\{\mathcal{T}_{h}\right\}_{h>0}$ we denote a family of regular triangulations of $\Omega$, built up using triangles $K$ with boundary $\partial K=F_{1} \cup F_{2} \cup F_{3}, h_{K}:=\operatorname{diam}(K)$ and $h:=\max \left\{h_{K}: K \in \mathcal{T}_{h}\right\}$.

We denote by $\mathcal{E}_{\Omega}$ the set of internal edges of $\mathcal{T}_{h}$ and for $K \in \mathcal{T}_{h}$ we denote by $\mathcal{E}(K)$ the set of its sides. The characteristic length of $F \in \mathcal{E}_{\Omega}$ is $h_{F}=|F|, \boldsymbol{n}$ is the normal outward vector on $\partial K, \partial_{\boldsymbol{s}}$ and $\partial_{\boldsymbol{n}}$ are the tangential and normal derivative operators, respectively, and $\mathbf{I}$ is the $\mathbb{R}^{2 \times 2}$ identity matrix. Also, for $K \in \mathcal{T}_{h}$ and $F \in \mathcal{E}_{\Omega}$ we define the following neighborhoods:

$$
\omega_{K}:=\cup\left\{K^{\prime} \in \mathcal{T}_{h}: K \cap K^{\prime} \neq \emptyset\right\} \quad, \quad \omega_{F}:=\cup\left\{K^{\prime} \in \mathcal{T}_{h}: F \in \mathcal{E}\left(K^{\prime}\right)\right\}
$$

For $F \in \mathcal{E}_{\Omega}$ we denote by $\llbracket v \rrbracket_{F}$ the jump of a function $v$ across $F$. Further, we introduce the standard linear finite element space

$$
V_{h}=\left\{v \in C^{0}(\bar{\Omega}):\left.v\right|_{K} \in \mathbb{P}_{1}(K), \forall K \in \mathcal{T}_{h}\right\}
$$

Finally, $H^{1}\left(\mathcal{T}_{h}\right)$ and $H_{0}^{1}\left(\mathcal{T}_{h}\right)$ stand for the spaces of functions whose restriction to $K \in \mathcal{T}_{h}$ belongs to $H^{1}(K)$ and $H_{0}^{1}(K)$, respectively, and we present a space $E_{h} \subset H^{1}\left(\mathcal{T}_{h}\right)$ (which, although not supposed finite dimensional, will turn out to be of finite dimension), called multiscale space, which will be used to enrich the trial space and will be problem dependent. Although we do not need a priori this space to satisfy $V_{h} \cap E_{h}=\{0\}$, this is a propery that the enrichment space ought to satisy.

\section{A review of Petrov-Galerkin Enriched Methods}

Petrov-Galerkin enriched methods (PGEM) are designed to give superior accuracy along with enhanced stability. The method is based on the variational formulation of a specific model and is obtained by approximating the trial function by piecewise polynomials enriched with multiscale functions; the test function is approximated by piecewise polynomials enriched with bubble functions. This difference between the approximations of the test and trial functions is part of the Petrov-Galerkin framework.

We have zero boundary conditions on element edges (or faces in 3D) by selecting bubbles as enrichment of test functions. This enables static condensation. As a result, a differential equation for the enrichment function holds for each element and the multiscale enrichment can be condensed as a function of the piecewise component of the solution and the data. Once the expression of the multiscale component of the solution is available we then substitute it 
into the equation tested by the piecewise polynomial component. The method that arises is a stabilized method with several improvements. Among these, we can quote;

- the enrichment produces an additional stability without compromising consistency in a different manner than standard stabilized methods;

- the accuracy is improved by letting the multiscale enrichment be different than zero on the element boundaries;

- the additional stabilizing terms may have a different form than the apparent canonical modifications using least-squares or adjoint operators.

The latter is the key on accuracy comparisons with stabilized methods.

We start our review by looking at the first PGEM in reactive-diffusive problems [22, 21]. Let us start by recalling the model: find $u$ such that

$$
\begin{aligned}
\sigma u-\triangle u=f & \text { in } \Omega, \\
u=0 & \text { on } \partial \Omega,
\end{aligned}
$$

where $\sigma \in \mathbb{R}^{+}$denotes the reactive constant and $f$ is a given datum. The usual variational formulation for this problem is given by: Find $u \in H_{0}^{1}(\Omega)$ such that:

$$
A(u, v)=(f, v)_{\Omega} \quad \forall v \in H_{0}^{1}(\Omega)
$$

where

$$
A(u, v):=\sigma(u, v)_{\Omega}+(\nabla u, \nabla v)_{\Omega}
$$

We take the trial enrichment to be in $E_{h}$ and the test enrichment to be in $H_{0}^{1}(K)$. These are enrichments to piecewise linear $u_{1}$ and $v_{1}$, respectively. The starting point towards the PGEM is then: find $u_{1}+u_{e} \in V_{h}+E_{h}$ such that

$$
A\left(u_{1}+u_{e}, v_{1}+v_{b}\right)=\left(f, v_{1}+v_{b}\right)_{\Omega} \quad \forall v_{1}+v_{b} \in V_{h} \oplus H_{0}^{1}\left(\mathcal{T}_{h}\right) .
$$

Considering $v_{1}=0$ we have an equation in each element as follows:

$$
\mathcal{L} u_{e}=-\sigma u_{1}+\Delta u_{1}+f=-\sigma u_{1}+f
$$

where we used the linearity of $u_{1}$ in $K$.

This problem needs a boundary condition. One possibility is to set zero as the boundary condition which would reduce the method to the residual-free-bubble method. We explore new possibilities to allow the enrichment to be non-zero on the boundary. For this particular 
model, and with the aim of correcting the residual of the strong equation on the boundary of $K$, in $[22,21]$ the following boundary condition is chosen for $u_{e}$ :

$$
\bar{\sigma} u_{e}-\partial_{s s} u_{e}=\frac{\bar{\sigma}}{\sigma}\left(f-\sigma u_{1}\right) \quad \text { along each } F \subseteq \partial K \text { and } u_{e}=0 \text { at the nodes, }
$$

where $\bar{\sigma}$ represents $\sigma$ multiplied by a suitable constant. This perturbation of $\sigma$ has been introduced to make it possible to compute the solution to this problem analytically.

Combining (6) and (7) we can solve for $\left.u_{e}\right|_{K}$ to get

$$
u_{e}=\mathcal{M}_{K}\left(f-\sigma u_{1}\right),
$$

in every $K \in \mathcal{T}_{h}$, where $\mathcal{M}_{K}$ is the solution operator associated to (6)-(7). This is then replaced in (5) to obtain the following PGEM method: Find $u_{1} \in V_{h}$ such that:

$$
A\left(u_{1}-\sigma \mathcal{M}_{K}\left(u_{1}\right), v_{1}\right)=\left(f, v_{1}\right)_{\Omega}-A\left(\mathcal{M}_{K}(f), v_{1}\right) \quad \forall v_{1} \in V_{h},
$$

which is a stabilized alike method, which has been proved to be well-posed in [21]. Note that (8) is a formal result that needs to be computed in detail. We do this by using basis functions for $u_{1}$ in the right-hand-sides of equations (6) and (7), and supposing that $f \in V_{h}$, which is an approximation that does not undermine the precision of the method (see [1] for the analysis in the case of a Stokes problem). For further details the interested reader is referred to $[22,21]$ and to [3] for an a posteriori error estimator. Finally, we note that the characterization (8) leads us to precisely define the space $E_{h}$ as follows

$$
E_{h}=\left\{v_{e} \in H_{0}^{1}\left(\mathcal{T}_{h}\right):\left.v_{e}\right|_{K}=\mathcal{M}_{K}\left(v_{1}\right), v_{1} \in V_{h}, \forall K \in \mathcal{T}_{h}\right\}
$$

and hence $E_{h}$ is of finite dimension and moreover, it clearly satisfies $E_{h} \cap V_{h}=\{0\}$.

Remark. We remark that the problem (5) may not be well-posed in its original version, but, once the boundary condition (7) is chosen, then the problem becomes well-posed.

Next, still keeping polynomial spaces enhanced with the solution of the local problem (8), a parabolic version of PGEM is proposed in [24] to deal with the unsteady reactiondiffusion problem. Stability is achieved for the reaction dominated case although persisting spurious oscillations show up as soon as small time step procedure is used. Consequently, it appears that overcoming such drawback demands replacing steady local enrichment (8) by its time-dependent version. In [32] this issue is addressed.

When applied to advection dominated problems, the PGEM aims to resolve internal and external exponential boundary layers. It stems from [23] that such cumbersome goal is accurately accomplished for external layers but not for internal ones since it is still highly mesh 
dependent. Therefore, it emerges from [23, 17] that compromising stability and flexibility leads to a non-conforming approach where the RFB method is adopted for internal elements while the PGEM is set for elements touching external boundaries of $\Omega$.

Turning back to mixed problems, a class of new stabilized finite element methods have been derived to tackle the Stokes model. Roughly, this is accomplished following through analogous steps as for the reaction-diffusion case, but now, just the velocity space is enhanced. Continuous piecewise linear space for the velocity and continuous piecewise linear or constant spaces for pressure have been made compatible in the sense of inf-sup condition by adding the multiscale function $\boldsymbol{u}_{e}$ to the linear contribution $\boldsymbol{u}_{1}$. The former solves the following elliptic problem

$$
-\nu \Delta \boldsymbol{u}_{e}=\boldsymbol{f}-\nabla p_{k}
$$

where $\nu \in \mathbb{R}^{+}$represents viscosity, $p_{k}$ the polynomial pressure variable with order $k=0,1$ and $\boldsymbol{f}$ is given datum. Concerning boundary conditions, however, we disregard the previous strategy and propose a quite different approach based on a posteriori error estimates. As a matter of fact, it can be shown that numerical errors are strongly related to the jumps of pressure and normal derivative of velocity on internal edges, and thus, we propose to correct them imposing the following boundary condition on $\boldsymbol{u}_{e}: \boldsymbol{u}_{e}=\mathbf{0}$ if $F \subset \partial \Omega$, else $\boldsymbol{u}_{e}$ solves

$$
\begin{aligned}
-\nu \partial_{\boldsymbol{s s}} \boldsymbol{u}_{e} & =\frac{1}{h_{F}} \llbracket \nu \partial_{\boldsymbol{n}} \boldsymbol{u}_{1} \pm p_{k} \mathbf{I} \cdot \boldsymbol{n} \rrbracket_{F} \quad \text { on } F \\
\boldsymbol{u}_{e} & =\mathbf{0} \text { at the nodes. }
\end{aligned}
$$

It can be proved that all the derived methods achieve optimal convergence [1] and lead, naturally, to a posteriori error estimators [2].

A second example of a mixed problem is the Darcy model. In its mixed form it presents an additional variable besides the pressure, the so-called Darcy velocity, which is proportional to the gradient of pressure. Unlike the Stokes case, now we ought to enrich both velocity and pressure spaces in order to make the continuous piecewise linear and constant spaces compatible, and even more important, to end up with locally mass conservative methods [7]. Going through the enriching methodology, it turns out that the piecewise linear velocity and the constant pressure $\left(\boldsymbol{u}_{1}, p_{0}\right)$ have to be element-wise augmented with the function $\left(\boldsymbol{u}_{e}, p_{e}\right)$ which solves the Darcy problem:

$$
\sigma \boldsymbol{u}_{e}+\nabla p_{e}=\boldsymbol{f}-\sigma \boldsymbol{u}_{1}, \quad \nabla \cdot \boldsymbol{u}_{e}=C_{K} \quad \text { in } K
$$


where $C_{K}$ is a suitable constant. Concerning the boundary condition for (11) two different alternatives have been undertaken in [7] (leading to fix $C_{K}$ ). First, following the idea used for the Stokes case we set the boundary condition for $\boldsymbol{u}_{e}$ as:

$$
\boldsymbol{u}_{e} \cdot \boldsymbol{n}=\frac{\alpha_{F} h_{F}}{\sigma} \llbracket p_{0} \rrbracket_{F} \quad \text { on each } F \subseteq \partial K \cap \Omega,
$$

where $\alpha_{F}$ is a positive constant close to one and independent of $h$ which can vary on each $F$. We point out that such choice keeps final methods conforming while stability is achieved without losing the local mass conservation feature. Alternatively, we can consider $\boldsymbol{u}_{e}$ satisfying

$$
\boldsymbol{u}_{e} \cdot \boldsymbol{n}=\frac{\alpha_{F} h_{F}}{\sigma} \llbracket p_{0} \rrbracket_{F}-\boldsymbol{u}_{1} \cdot \boldsymbol{n}+\frac{1}{h_{F}} \int_{F} \boldsymbol{u}_{1} \cdot \boldsymbol{n} \quad \text { on each } F \subseteq \partial K \cap \Omega .
$$

This second choice mixes the strategy of [22] and [1] and preserves all desirable properties of (12). Furthermore, analytical solutions arise easily avoiding additional computational costs due to two level calculations.

Applying the technique described above, in the next section we derive a new stabilized finite element method for the generalized Stokes problem taking care of the inf-sup condition and the boundary layer issue simultaneously.

\section{An Applichtion to the Generalized Stokes Problem}

Let $\boldsymbol{f} \in L^{2}(\Omega)^{2}$ and let us consider the following generalized Stokes problem: Find $(\boldsymbol{u}, p)$ such that

$$
\begin{aligned}
\mathcal{L} \boldsymbol{u}+\nabla p=\boldsymbol{f}, & \nabla \cdot \boldsymbol{u}=0 \quad \text { in } \Omega \\
\boldsymbol{u}=\mathbf{0} & \text { on } \partial \Omega,
\end{aligned}
$$

where $\mathcal{L} \boldsymbol{u}:=\sigma \boldsymbol{u}-\nu \Delta \boldsymbol{u}$, and we recall that $\sigma, \nu \in \mathbb{R}^{+}$denote the reaction term and the fluid viscosity, respectively. The usual variational formulation for problem (14) is given by: Find $(\boldsymbol{u}, p) \in \mathbf{V} \times Q:=H_{0}^{1}(\Omega)^{2} \times L_{0}^{2}(\Omega)$ such that:

$$
\mathbf{B}((\boldsymbol{u}, p),(\boldsymbol{v}, q))=\mathbf{F}(\boldsymbol{v}, q) \quad \forall(\boldsymbol{v}, q) \in \mathbf{V} \times Q
$$

where

$$
\begin{gathered}
\mathbf{B}((\boldsymbol{u}, p),(\boldsymbol{v}, q)):=\sigma(\boldsymbol{u}, \boldsymbol{v})_{\Omega}+\nu(\nabla \boldsymbol{u}, \nabla \boldsymbol{v})_{\Omega}-(p, \nabla \cdot \boldsymbol{v})_{\Omega}+(q, \nabla \cdot \boldsymbol{u})_{\Omega} \\
\mathbf{F}(\boldsymbol{v}, q):=(\boldsymbol{f}, \boldsymbol{v})_{\Omega}
\end{gathered}
$$


Hereafter, we will define the bilinear form $a(.,$.$) over \mathbf{V} \times \mathbf{V}$ by

$$
a(\boldsymbol{u}, \boldsymbol{v}):=\sigma(\boldsymbol{u}, \boldsymbol{v})_{\Omega}+\nu(\nabla \boldsymbol{u}, \nabla \boldsymbol{v})_{\Omega} .
$$

In order to propose the Petrov-Galerkin method for (14), let $\mathbf{V}_{h}:=\left[V_{h} \cap H_{0}^{1}(\Omega)\right]^{2}, Q_{h}:=$ $V_{h} \cap L_{0}^{2}(\Omega)$, where $V_{h}$ is defined in (1). Then, we propose the following scheme for (14): Find $\boldsymbol{u}_{1}+\boldsymbol{u}_{e} \in \mathbf{V}_{h}+\left[E_{h}\right]^{2}$ and $p_{1} \in Q_{h}$ such that

$$
\mathbf{B}\left(\left(\boldsymbol{u}_{1}+\boldsymbol{u}_{e}, p_{1}\right),\left(\boldsymbol{v}_{1}+\boldsymbol{v}_{b}, q_{1}\right)\right)=\mathbf{F}\left(\boldsymbol{v}_{1}+\boldsymbol{v}_{b}, q_{1}\right),
$$

for all $\boldsymbol{v}_{1}+\boldsymbol{v}_{b} \in \mathbf{V}_{h} \oplus\left[H_{0}^{1}\left(\mathcal{T}_{h}\right)\right]^{2}$ and all $q_{1} \in Q_{h}$. This Petrov-Galerkin scheme may be written as the following system:

$$
\begin{array}{cc}
\mathbf{B}\left(\left(\boldsymbol{u}_{1}+\boldsymbol{u}_{e}, p_{1}\right),\left(\boldsymbol{v}_{1}, q_{1}\right)\right)=\mathbf{F}\left(\boldsymbol{v}_{1}, q_{1}\right) & \forall\left(\boldsymbol{v}_{1}, q_{1}\right) \in \mathbf{V}_{h} \times Q_{h}, \\
a\left(\boldsymbol{u}_{1}+\boldsymbol{u}_{e}, \boldsymbol{v}_{b}\right)_{K}-\left(p_{1}, \nabla \cdot \boldsymbol{v}_{b}\right)_{K}=\left(\boldsymbol{f}, \boldsymbol{v}_{b}\right)_{K} & \forall \boldsymbol{v}_{b} \in H_{0}^{1}(K)^{2}, \forall K \in \mathcal{T}_{h},
\end{array}
$$

where the subindex $K$ stands for integration over $K$. Equation (20) above may be written in strong form in the following way

$$
\mathcal{L} \boldsymbol{u}_{e}=\boldsymbol{f}-\left(\sigma \boldsymbol{u}_{1}+\nabla p_{1}\right) \quad \text { in } K .
$$

From now on, and just for the derivation of the method, we will suppose that $\boldsymbol{f} \in\left[V_{h}\right]^{2}$. Now, this differential problem above must be completed with boundary conditions. In order to correct also the residual of the strong equation on the boundary of $K$, we impose the following boundary condition on $\boldsymbol{u}_{e}$ :

$$
\boldsymbol{u}_{e}=\boldsymbol{g}_{e} \quad \text { on } F_{i}, \quad i=1,2,3,
$$

where $\boldsymbol{g}_{e}$ will appear as solution of a suitable ODE, with right-hand side depending on $\boldsymbol{f}, \boldsymbol{u}_{1}$ and $p_{1}$, on each edge $F_{i}$ (this ODE will be specified, for the basis functions, in $\S 3.1$ below). Since this problem is well posed, we can write (19) as follows: Find $\left(\boldsymbol{u}_{1}, p_{1}\right) \in \mathbf{V}_{h} \times Q_{h}$ such that

$$
\sum_{K \in \mathcal{T}_{h}}\left[a\left(\boldsymbol{u}_{1}+\boldsymbol{u}_{e}^{K}, \boldsymbol{v}_{1}\right)_{K}-\left(p_{1}, \nabla \cdot \boldsymbol{v}_{1}\right)_{K}+\left(q_{1}, \nabla \cdot\left(\boldsymbol{u}_{1}+\boldsymbol{u}_{e}^{K}\right)\right)_{K}\right]=\left(\boldsymbol{f}, \boldsymbol{v}_{1}\right)_{\Omega},
$$

for all $\left(\boldsymbol{v}_{1}, q_{1}\right) \in \mathbf{V}_{h} \times Q_{h}$, where $\boldsymbol{u}_{e}^{K}:=\left.\boldsymbol{u}_{e}\right|_{K}$. Next, in order to give a more practical (and useful in the sequel) formulation, we define, as in (8), an operator $\mathcal{M}_{K}: \mathbb{P}_{1}(K)^{2} \rightarrow H^{1}(K)^{2}$ such that

$$
\boldsymbol{u}_{e}^{K}=\mathcal{M}_{K}\left(\boldsymbol{f}-\sigma \boldsymbol{u}_{1}-\nabla p_{1}\right) \quad \forall K \in \mathcal{T}_{h}
$$


Thus, with the characterization (24), the problem (19) leads to the following Petrov-Galerkin Enriched Method (PGEM): Find $\left(\boldsymbol{u}_{1}, p_{1}\right) \in \mathbf{V}_{h} \times Q_{h}$ such that

$$
\begin{gathered}
\mathbf{B}_{m}\left(\left(\boldsymbol{u}_{1}, p_{1}\right),\left(\boldsymbol{v}_{1}, q_{1}\right)\right):= \\
\sum_{K \in \mathcal{T}_{h}}\left[a\left(\boldsymbol{u}_{1}-\mathcal{M}_{K}\left(\sigma \boldsymbol{u}_{1}+\nabla p_{1}\right), \boldsymbol{v}_{1}\right)_{K}-\left(p_{1}, \nabla \cdot \boldsymbol{v}_{1}\right)_{K}+\left(q_{1}, \nabla \cdot\left(\boldsymbol{u}_{1}-\mathcal{M}_{K}\left(\sigma \boldsymbol{u}_{1}+\nabla p_{1}\right)\right)\right)_{K}\right] \\
=\left(\boldsymbol{f}, \boldsymbol{v}_{1}\right)_{\Omega}-\sum_{K \in \mathcal{T}_{h}}\left[a\left(\mathcal{M}_{K} \boldsymbol{f}, \boldsymbol{v}_{1}\right)_{K}-\left(q_{1}, \nabla \cdot\left(\mathcal{M}_{K} \boldsymbol{f}\right)\right)_{K}\right],
\end{gathered}
$$

for all $\left(\boldsymbol{v}_{1}, q_{1}\right) \in \mathbf{V}_{h} \times Q_{h}$.

3.1. The basis functions. We describe now the way of implementing (25) in terms of the basis functions. Let $\psi_{1}, \psi_{2}, \psi_{3}$ denote the barycentric coordinates of the element $K$. We enumerate the sides $F_{i}, i=1,2,3$ such that $\left.\psi_{i}\right|_{F_{i}}=0$ and define as $b_{K}^{i}$ the solution of

$$
\begin{aligned}
\mathcal{L} b_{K}^{i} & =\psi_{i} \text { in } K \\
\text { for } j=1,2,3: \quad \overline{\boldsymbol{\sigma}}_{j}^{i} b_{K}^{i}-\nu \partial_{\boldsymbol{s s}} b_{K}^{i} & =\frac{\overline{\boldsymbol{\sigma}}_{j}^{i}}{\sigma} \psi_{i} \quad \text { on } F_{j}, \quad b_{K}^{i}=0 \quad \text { at the nodes },
\end{aligned}
$$

where, suggested by [22], we have made the choice

$$
\overline{\boldsymbol{\sigma}}_{j}^{i}=\sigma \frac{4|K|^{2}}{\left|F_{j}\right|^{2}\left|F_{i}\right|^{2}}
$$

The local problem can be solved analytically, obtaining

$$
b_{K}^{i}(x, y)=\frac{1}{\sigma}\left(\psi_{i}(x, y)-\frac{\sinh \left(\alpha_{i} \psi_{i}\right)}{\sinh \left(\alpha_{i}\right)}\right) \quad \text { where } \quad \alpha_{i}=\sqrt{\frac{4 \sigma|K|^{2}}{\nu\left|F_{i}\right|^{2}}},
$$

and hence, we see that, for a linear function $\boldsymbol{g}=\left(g_{1}, g_{2}\right)=\left(\sum_{i=1}^{3} g_{1}^{i} \psi_{i}, \sum_{j=1}^{3} g_{2}^{j} \psi_{j}\right)$, we have that the operator $\mathcal{M}_{K}$ defined in (24) is given by

$$
\mathcal{M}_{K}(\boldsymbol{g})=\left(\sum_{i=1}^{3} g_{1}^{i} b_{K}^{i}, \sum_{j=1}^{3} g_{2}^{j} b_{K}^{j}\right)
$$

Hence, an exact expression for the basis functions to be used in the implementation of (25) is available, thus leading to a method which is not of a two level type. Finally, we note that we can exactly formulate the enriched space $E_{h}$ as the sub-space of $H^{1}\left(\mathcal{T}_{h}\right)$ whose functions are locally linear combinations of the functions $b_{K}^{i}$, and hence it is again finite dimensional. 
Remark. Let $b_{K}=\sum_{i=1}^{3} b_{K}^{i}$, i.e.,

$$
b_{K}(x, y)=\frac{1}{\sigma}\left(1-\sum_{i=1}^{3} \frac{\sinh \left(\alpha_{i} \psi_{i}\right)}{\sinh \left(\alpha_{i}\right)}\right)
$$

In Figure 1 we depict the function $b_{K}$ in a patch of equilateral elements for different values of $\alpha_{K}:=\alpha_{1}=\alpha_{2}=\alpha_{3}$. In there we can appreciate how this function varies with respect to $\alpha_{K}$. This will have a direct impact on the error analysis performed in Section 3.3. We can also compute the mean value of $b_{K}$ on $K$, which will be very useful in the definition of our stabilization parameter (see (36) below). Indeed, from the expression for $b_{K}$ we obtain

$$
\frac{\left(b_{K}, 1\right)_{K}}{|K|}=\frac{1}{\sigma}\left[1-2 \sum_{i=1}^{3}\left(\frac{1}{\alpha_{i}^{2}}-\frac{1}{\alpha_{i} \sinh \left(\alpha_{i}\right)}\right)\right] \text {. }
$$

We further remark that, in the case where the mesh $\mathcal{T}_{h}$ is composed by equilateral triangles, then $\overline{\boldsymbol{\sigma}}_{j}^{i}=\overline{\boldsymbol{\sigma}}$ for $i, j=1,2,3$, and then $b_{K}$ satisfies the following boundary value problem in $K$ :

$$
\mathcal{L} b_{K}=1 \quad \text { in } K, \quad b_{K}=g \quad \text { on } \partial K
$$

where, for $i=1,2,3$,

$$
\overline{\boldsymbol{\sigma}} g-\nu \partial_{\boldsymbol{s s}} g=\frac{\overline{\boldsymbol{\sigma}}}{\sigma} \quad \text { on } F_{i}, \quad g=0 \quad \text { at the nodes } .
$$

Finally, using these functions, we may now give a precise definition of the function $\boldsymbol{g}_{e}$ appearing in (22). Indeed, we have

$$
\boldsymbol{g}_{e}=\left(\sum_{i=1}^{3}\left(f_{i}^{1}-\sigma u_{i}^{1}\right) b_{K}^{i}-b_{K} \frac{\partial p_{1}}{\partial x_{1}}, \sum_{j=1}^{3}\left(f_{j}^{2}-\sigma u_{j}^{2}\right) b_{K}^{j}-b_{K} \frac{\partial p_{1}}{\partial x_{2}}\right)
$$

where $f_{i}^{k}, u_{i}^{k}, k=1,2, i=1,2,3$, stand for the nodal values of $\boldsymbol{f}$ and $\boldsymbol{u}_{1}$, respectively.

Remark. We end this section by returning to the method (25). Since both the unknowns $\left(\boldsymbol{u}_{1}, p_{1}\right)$ and the test functions $\left(\boldsymbol{v}_{1}, q_{1}\right)$ belong to the same space, (25) could be seen as a Galerkin method. We remark nevertheless that it can be seen alternatively as a PetrovGalerkin method. Indeed, the solution of (25) may be also given by $\boldsymbol{u}_{1}+\boldsymbol{u}_{e}=\boldsymbol{u}_{1}+\mathcal{M}_{K}(\boldsymbol{f}-$ $\left.\sigma \boldsymbol{u}_{1}-\nabla p_{1}\right) \in \mathbf{V}_{h} \oplus\left[E_{h}\right]^{2}$, and hence the discrete solution belongs to a space different from the test space, hence, the Petrov-Galerkin character. 

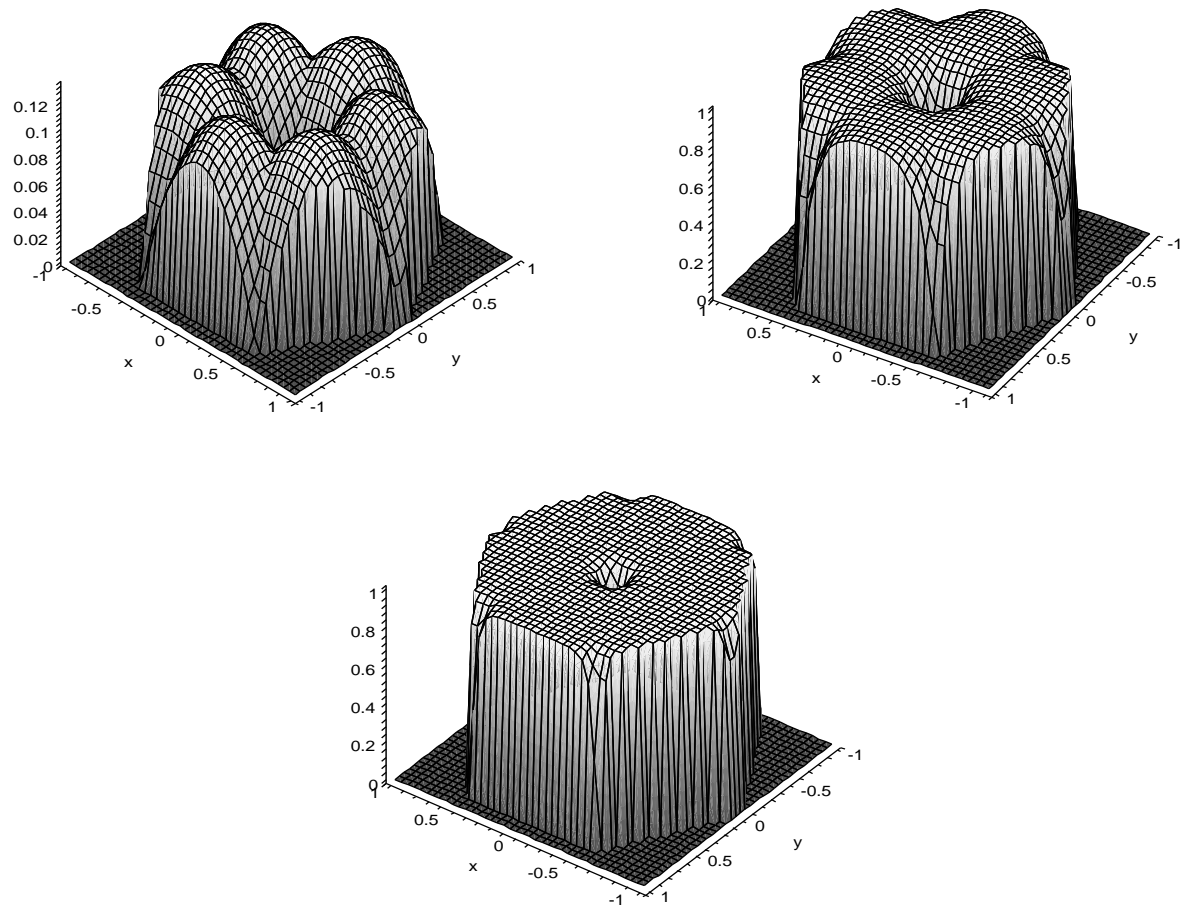

Figure 1. Shape of $\sigma b_{K}$ on a patch of elements with $\alpha_{i}=1, \alpha_{i}=10$ and $\alpha_{i}=25$.

3.2. A link to a stabilized formulation. We begin by presenting the stabilized finite element method: Find $\left(\boldsymbol{u}_{1}, p_{1}\right) \in \mathbf{V}_{h} \times Q_{h}$ such that

$$
\mathbf{B}_{\tau}\left(\left(\boldsymbol{u}_{1}, p_{1}\right),\left(\boldsymbol{v}_{1}, q_{1}\right)\right)=\mathbf{F}_{\tau}\left(\boldsymbol{v}_{1}, q_{1}\right) \quad \forall\left(\boldsymbol{v}_{1}, q_{1}\right) \in \mathbf{V}_{h} \times Q_{h},
$$

where

$$
\begin{gathered}
\mathbf{B}_{\tau}\left(\left(\boldsymbol{u}_{1}, p_{1}\right),\left(\boldsymbol{v}_{1}, q_{1}\right)\right):=\mathbf{B}\left(\left(\boldsymbol{u}_{1}, p_{1}\right),\left(\boldsymbol{v}_{1}, q_{1}\right)\right)-\sum_{K \in \mathcal{T}_{h}} \tau_{K}\left(\sigma \boldsymbol{u}_{1}+\nabla p_{1}, \sigma \boldsymbol{v}_{1}-\nabla q_{1}\right)_{K}, \\
\mathbf{F}_{\tau}\left(\boldsymbol{v}_{1}, q_{1}\right):=\mathbf{F}\left(\boldsymbol{v}_{1}, q_{1}\right)-\sum_{K \in \mathcal{T}_{h}} \tau_{K}\left(\boldsymbol{f}, \sigma \boldsymbol{v}_{1}-\nabla q_{1}\right)_{K},
\end{gathered}
$$

and the stabilization parameter is given by

$$
\tau_{K}:=\frac{1}{\sigma}\left[1-2 \sum_{i=1}^{3}\left(\frac{1}{\alpha_{i}^{2}}-\frac{1}{\alpha_{i} \sinh \left(\alpha_{i}\right)}\right)\right] .
$$

Remark. Method (35) has some similarities with some existing stabilized finite element methods for this problem, specially with [8] and [6]. The are two main differences between the present method and the method presented in [6]. The first one is related to the extension to 
higher order polynomials. Even if this work is focused in first order approximations, an extension to arbitrary order seems feasible. In that case, the resulting formulation would have a structure similar to the unusual stabilized finite element methods from [8], thus having a symmetric formulation, contrary to the method from [6] in which the formulation is nonsymmetric for higher degrees of interpolation. Concerning the stabilization parameter, the main difference relies on the fact that in the present approach the stabilization parameter is exactly known as being the mean value of the enrichment function on the element, contrary to a "virtual" (not known) bubble in [8] and an ad-hoc expression arising from the stability analysis in [6].

3.2.1. Derivation. For completeness of the presentation, we resume the derivation carried out in [9]. We will suppose that the mesh $\mathcal{T}_{h}$ is made by equilateral triangles. The first step is to replace in our formulation $\boldsymbol{u}_{e}$ by

$$
\tilde{\boldsymbol{u}}_{e}:=\mathcal{M}_{K}\left(\boldsymbol{f}-\sigma \overline{\boldsymbol{u}}_{1}-\nabla p_{1}\right)=b_{K}\left(\boldsymbol{f}-\sigma \overline{\boldsymbol{u}}_{1}-\nabla p_{1}\right)
$$

where, for a function $v, \bar{v}$ denotes its projection onto the $\mathbb{P}_{0}(K)$ space, i.e.,

$$
\bar{v}:=\frac{(v, 1)_{K}}{|K|} .
$$

We further remark that $b_{K}$ satisfies

$$
\left\|b_{K}\right\|_{0, K} \leq C h_{K}^{3} \quad \text { and } \quad\left\|b_{K}\right\|_{0, \partial K} \leq C h_{K}^{5 / 2}
$$

where $C>0$ is a positive constant depending possibly on $\sigma$ and $\nu$, but independent of $h$.

Next, in order to design a stabilized finite element method we integrate by parts and arrive at the following rewriting of (19) (or (25)):

$$
\mathbf{B}\left(\left(\boldsymbol{u}_{1}, p_{1}\right),\left(\boldsymbol{v}_{1}, q_{1}\right)\right)+\sum_{K \in \mathcal{T}_{h}}\left[\left(\tilde{\boldsymbol{u}}_{e}, \sigma \boldsymbol{v}_{1}-\nabla q_{1}\right)_{K}+\left(\tilde{\boldsymbol{u}}_{e}, \nu \partial_{\boldsymbol{n}} \boldsymbol{v}_{1}+q_{1} \mathbf{I} \cdot \boldsymbol{n}\right)_{\partial K}\right]=\mathbf{F}\left(\boldsymbol{v}_{1}, q_{1}\right) \text {. }
$$

Next, we neglect the boundary terms (see [9] for a discussion about this matter). Also, using (38) and the approximation properties of the projection (cf. [19]), we obtain

$$
\sum_{K \in \mathcal{T}_{h}}\left(\tilde{\boldsymbol{u}}_{e}, \sigma\left(\boldsymbol{v}_{1}-\overline{\boldsymbol{v}}_{1}\right)\right)_{K} \leq C h_{K}^{3}\left\|\boldsymbol{f}-\sigma \overline{\boldsymbol{u}}_{1}-\nabla p_{1}\right\|_{0, K}\left|\boldsymbol{v}_{1}\right|_{1, K}
$$

and hence, using (37) and the orthogonality of the projection, the following approximation is justified

$$
\sum_{K \in \mathcal{T}_{h}}\left(\tilde{\boldsymbol{u}}_{e}, \sigma \boldsymbol{v}_{1}-\nabla q_{1}\right)_{K} \approx \sum_{K \in \mathcal{T}_{h}} \frac{\left(b_{K}, 1\right)_{K}}{|K|}\left(\boldsymbol{f}-\sigma \overline{\boldsymbol{u}}_{1}-\nabla p_{1}, \sigma \boldsymbol{v}_{1}-\nabla q_{1}\right)_{K}
$$


Collecting all the previous results, we can present the following stabilized finite element method for (14): Find $\left(\boldsymbol{u}_{1}, p_{1}\right) \in \mathbf{V}_{h} \times Q_{h}$ such that

$$
\begin{gathered}
\mathbf{B}\left(\left(\boldsymbol{u}_{1}, p_{1}\right),\left(\boldsymbol{v}_{1}, q_{1}\right)\right)-\sum_{K \in \mathcal{T}_{h}} \frac{\left(b_{K}, 1\right)_{K}}{|K|}\left(\sigma \overline{\boldsymbol{u}}_{1}+\nabla p_{1}, \sigma \boldsymbol{v}_{1}-\nabla q_{1}\right)_{K} \\
=\mathbf{F}\left(\boldsymbol{v}_{1}, q_{1}\right)-\sum_{K \in \mathcal{T}_{h}} \frac{\left(b_{K}, 1\right)_{K}}{|K|}\left(\boldsymbol{f}, \sigma \boldsymbol{v}_{1}-\nabla q_{1}\right)_{K}
\end{gathered}
$$

for all $\left(\boldsymbol{v}_{1}, q_{1}\right) \in \mathbf{V}_{h} \times Q_{h}$. We finally remark that, replacing the added terms in $K$ by $\left(\sigma \boldsymbol{u}_{1}+\nabla p_{1}, \sigma \boldsymbol{v}_{1}-\nabla q_{1}\right)_{K}$ (which introduces a new source of error, but, again, this error is of a smaller size), and noting that $\frac{\left(b_{K}, 1\right)_{K}}{|K|}$ is equal to $\tau_{K}$, then method (39) is nothing but method (35).

3.3. Convergence analysis and error estimates. This section is devoted to the a priori error analysis of the method (35). We will start by giving a technical result concerning the properties of the stabilization parameter $\tau_{K}$ and then we will give a stability result for (35).

Lemma 1. Let $K \in \mathcal{T}_{h}$, let $\alpha_{K}=\max \left\{\alpha_{i}: i=1,2,3\right\}$ and $F \in \mathcal{E}(K)$ and $\omega_{F}=K \cup K^{\prime}$. Then, the following estimates hold for $\tau_{K}$ :

$$
\begin{aligned}
C_{1} \min \left\{1, \alpha_{K}^{2}\right\} & \leq \sigma \tau_{K} \leq C_{2} \min \left\{1, \alpha_{K}^{2}\right\}, \\
\frac{C_{1}}{1+\alpha_{K}^{2}} & \leq 1-\sigma \tau_{K} \leq \frac{C_{2}}{1+\alpha_{K}^{2}}, \\
\left|\llbracket 1-\sigma \tau_{K} \rrbracket_{F}\right| & \leq C \min \left\{1, \alpha_{K}^{2}\right\}
\end{aligned}
$$

where the (positive) constans $C, C_{1}$ and $C_{2}$ do not depend on $h, \sigma$ or $\nu$.

Proof. The results can be proved using a Taylor series expansion for the function $\sinh (\cdot)$, the definition of $\tau_{K}$ and the regularity of the mesh.

Next, let us define the following mesh-dependent norm:

$$
\|(\boldsymbol{v}, q)\|_{h}^{2}:=\sum_{K \in \mathcal{T}_{h}}\left[\sigma\left(1-\sigma \tau_{K}\right)\|\boldsymbol{v}\|_{0, K}^{2}+\nu|\boldsymbol{v}|_{1, K}^{2}+\tau_{K}\|\nabla q\|_{0, K}^{2}\right]
$$


Lemma 2. For all $(\boldsymbol{v}, q) \in\left[H^{1}(\Omega)\right]^{2} \times H^{1}(\Omega)$, the bilinear form $\mathbf{B}_{\tau}$ satisfies

$$
\begin{aligned}
\mathbf{B}_{\tau}((\boldsymbol{v}, q),(\boldsymbol{v}, q))= & \|(\boldsymbol{v}, q)\|_{h}^{2}, \\
\mathbf{B}_{\tau}((\boldsymbol{v}, q),(\boldsymbol{w}, r)) \leq & \|(\boldsymbol{v}, q)\|_{h}\|(\boldsymbol{w}, r)\|_{h}+\sum_{K \in \mathcal{T}_{h}}\left(1-\sigma \tau_{K}\right)(\nabla q, \boldsymbol{w})_{K} \\
& +\sum_{K \in \mathcal{T}_{h}}\left(1-\sigma \tau_{K}\right)(\nabla \cdot \boldsymbol{v}, r)_{K}-\sum_{F \in \mathcal{E}_{\Omega}} \llbracket \sigma \tau_{K} \rrbracket_{F}(\boldsymbol{v}, r)_{F},
\end{aligned}
$$

and the discrete problem (35) has a unique solution.

Proof. The first equality follows easily from the definition of $\mathbf{B}_{\tau}$. The second one is straightfoward from the definition of $\mathbf{B}_{\tau}$, integration by parts and the Cauchy-Schwarz inequality.

The method (35) is not strongly consistent. Hence, we bound the consistency error in the following result.

Lemma 3. Let us suppose that $(\boldsymbol{u}, p) \in\left[H^{2}(\Omega) \cap H_{0}^{1}(\Omega)\right]^{2} \times\left[H^{1}(\Omega) \cap L_{0}^{2}(\Omega)\right]$. Then, there exists $C>0$ such that

$$
\mathbf{B}_{\tau}\left(\left(\boldsymbol{u}-\boldsymbol{u}_{1}, p-p_{1}\right),\left(\boldsymbol{v}_{1}, q_{1}\right)\right) \leq \sum_{K \in \mathcal{T}_{h}} \tau_{K} \nu\left(\Delta \boldsymbol{u}, \sigma \boldsymbol{v}_{1}\right)_{K}+C h \sqrt{\nu}|\boldsymbol{u}|_{2, \Omega}\left\|\left(\boldsymbol{v}_{1}, q_{1}\right)\right\|_{h}
$$

Proof. A simple computation shows that

$$
\mathbf{B}_{\tau}\left(\left(\boldsymbol{u}-\boldsymbol{u}_{1}, p-p_{1}\right),\left(\boldsymbol{v}_{1}, q_{1}\right)\right)=\sum_{K \in \mathcal{T}_{h}} \tau_{K}\left(\nu \Delta \boldsymbol{u}, \sigma \boldsymbol{v}_{1}-\nabla q_{1}\right)_{K}
$$

and the result follows from (40), the Cauchy-Schwarz inequality and the definition of $\|.\|_{h}$.

Remark. As it was done in [1] for the Stokes problem, we could have given a consistent presentation of our method, just keeping the $\Delta \boldsymbol{u}$ and $\Delta \boldsymbol{v}$ terms that vanish in the integration by parts appearing in the static condensation procedure from last section. In that case, the proof of Lemma 4 below would change since Lemma 2 should be written differently (since the modified bilinear form $\mathbf{B}_{\tau}$ would not be elliptic in the whole continuous space), but the results would be essentially the same.

Lemma 4. Let us suppose that $(\boldsymbol{u}, p) \in\left[H^{2}(\Omega) \cap H_{0}^{1}(\Omega)\right]^{2} \times\left[H^{1}(\Omega) \cap L_{0}^{2}(\Omega)\right]$ is the solution of (14) and that $\left(\boldsymbol{u}_{1}, p_{1}\right) \in \mathbf{V}_{h} \times Q_{h}$ is the solution of (35), and let us denote $\left(\boldsymbol{e}_{\boldsymbol{u}}, e_{p}\right):=$ 
$\left(\boldsymbol{u}-\boldsymbol{u}_{1}, p-p_{1}\right)$. Then, there exists $C>0$, independent of $h, \sigma$ and $\nu$, such that

$$
\begin{gathered}
\left\|\left(\boldsymbol{e}_{\boldsymbol{u}}, e_{p}\right)\right\|_{h}^{2} \leq C \inf _{\left(\boldsymbol{v}_{1}, q_{1}\right) \in \mathbf{V}_{h} \times Q_{h}}\left\{\left\|\left(\boldsymbol{u}-\boldsymbol{v}_{1}, p-q_{1}\right)\right\|_{h}^{2}+\sum_{K \in \mathcal{T}_{h}} \nu h_{K}^{-2}\left\|\boldsymbol{u}-\boldsymbol{v}_{1}\right\|_{0, K}^{2}+\nu h^{2}|\boldsymbol{u}|_{2, \Omega}^{2}\right. \\
\left.+\sum_{K \in \mathcal{T}_{h}} \frac{\min \left\{1, \alpha_{K}^{2}\right\}}{\sigma}\left(h_{K}^{-2}\left\|p-q_{1}\right\|_{0, K}^{2}+\left|p-q_{1}\right|_{1, K}^{2}\right)\right\} .
\end{gathered}
$$

Proof. Let $\left(\boldsymbol{v}_{1}, q_{1}\right) \in \mathbf{V}_{h} \times Q_{h}$. Then, from Lemmas 2 and 3 there follows

$$
\begin{aligned}
\left\|\left(\boldsymbol{e}_{\boldsymbol{u}}, e_{p}\right)\right\|_{h}^{2}= & \mathbf{B}_{\tau}\left(\left(\boldsymbol{e}_{\boldsymbol{u}}, e_{p}\right),\left(\boldsymbol{e}_{\boldsymbol{u}}, e_{p}\right)\right) \\
= & \mathbf{B}_{\tau}\left(\left(\boldsymbol{e}_{\boldsymbol{u}}, e_{p}\right),\left(\boldsymbol{u}-\boldsymbol{v}_{1}, p-q_{1}\right)\right)+\mathbf{B}_{\tau}\left(\left(\boldsymbol{e}_{\boldsymbol{u}}, e_{p}\right),\left(\boldsymbol{v}_{1}-\boldsymbol{u}_{1}, q_{1}-p_{1}\right)\right) \\
\leq & \left\|\left(\boldsymbol{e}_{\boldsymbol{u}}, e_{p}\right)\right\|_{h}\left\|\left(\boldsymbol{u}-\boldsymbol{v}_{1}, p-q_{1}\right)\right\|_{h}+\underbrace{\sum_{K \in \mathcal{T}_{h}}\left(1-\sigma \tau_{K}\right)\left(\nabla e_{p},\left(\boldsymbol{u}-\boldsymbol{v}_{1}\right)\right)_{K}}_{I} \\
& +\underbrace{+\sum_{K \in \mathcal{T}_{h}}\left(1-\sigma \tau_{K}\right)\left(\nabla \cdot \boldsymbol{e}_{\boldsymbol{u}},\left(p-q_{1}\right)\right)_{K}-\sum_{F \in \mathcal{E}_{\Omega}} \llbracket \sigma \tau_{K} \rrbracket_{F}\left(\boldsymbol{e}_{\boldsymbol{u}} \cdot \boldsymbol{n}, p-q_{1}\right)_{F}}_{I I} \\
& \underbrace{\sum_{K \in \mathcal{T}_{h}} \tau_{K}\left(\nu \Delta \boldsymbol{u}, \sigma\left(\boldsymbol{v}_{1}-\boldsymbol{u}_{1}\right)\right)_{K}}_{I I I}+C h \sqrt{\nu}|\boldsymbol{u}|_{2, \Omega}\left\|\left(\boldsymbol{v}_{1}-\boldsymbol{u}_{1}, q_{1}-p_{1}\right)\right\|_{h} .
\end{aligned}
$$

Now, we proceed term by term. First, using the Cauchy-Schwarz inequality we arrive at

$$
\begin{aligned}
I & =\sum_{K \in \mathcal{T}_{h}}\left(1-\sigma \tau_{K}\right)\left(\nabla e_{p}, \boldsymbol{u}-\boldsymbol{v}_{1}\right)_{K} \\
& \leq \sum_{K \in \mathcal{T}_{h}}\left(1-\sigma \tau_{K}\right)\left\|\nabla e_{p}\right\|_{0, K}\left\|\boldsymbol{u}-\boldsymbol{v}_{1}\right\|_{0, K} \\
& \leq C\left\{\sum_{K \in \mathcal{T}_{h}} \frac{\tau_{K}^{-1}}{1+\alpha_{K}^{2}}\left\|\boldsymbol{u}-\boldsymbol{v}_{1}\right\|_{0, K}^{2}\right\}^{\frac{1}{2}}\left\|\left(\boldsymbol{e}_{\boldsymbol{u}}, e_{p}\right)\right\|_{h} \\
& \leq C\left\{\sum_{K \in \mathcal{T}_{h}} \sigma \min \left\{1, \alpha_{K}^{-2}\right\} \max \left\{1, \alpha_{K}^{-2}\right\}\left\|\boldsymbol{u}-\boldsymbol{v}_{1}\right\|_{0, K}^{2}\right\}^{\frac{1}{2}}\left\|\left(\boldsymbol{e}_{\boldsymbol{u}}, e_{p}\right)\right\|_{h} \\
& =C\left\{\sum_{K \in \mathcal{T}_{h}} \sigma \alpha_{K}^{-2}\left\|\boldsymbol{u}-\boldsymbol{v}_{1}\right\|_{0, K}^{2}\right\}^{\frac{1}{2}}\left\|\left(\boldsymbol{e}_{\boldsymbol{u}}, e_{p}\right)\right\|_{h},
\end{aligned}
$$


where we have also used the fact that $1-\sigma \tau_{K} \leq 1,(40)$, (41) and the definition of the norm $\|\cdot\|_{h}$. Also, applying (41) we obtain

$$
\begin{aligned}
I I & =\sum_{K \in \mathcal{T}_{h}}\left(1-\sigma \tau_{K}\right)\left(p-q_{1}, \nabla \cdot \boldsymbol{e}_{\boldsymbol{u}}\right)_{K}-\sum_{F \in \mathcal{E}_{\Omega}} \llbracket \sigma \tau_{K} \rrbracket_{F}\left(\boldsymbol{e}_{\boldsymbol{u}} \cdot \boldsymbol{n}, p-q_{1}\right)_{F} \\
& \leq \sum_{K \in \mathcal{T}_{h}} \frac{C}{1+\alpha_{K}^{2}}\left\|p-q_{1}\right\|_{0, K}\left|\boldsymbol{e}_{\boldsymbol{u}}\right|_{1, K}+\sum_{F \in \mathcal{E}_{\Omega}}\left|\llbracket \sigma \tau_{K} \rrbracket_{F}\right|\left\|\boldsymbol{e}_{\boldsymbol{u}}\right\|_{0, F}\left\|p-q_{1}\right\|_{0, F} \\
& \leq C\left\{\sum_{K \in \mathcal{T}_{h}} \frac{\nu^{-1}}{1+\alpha_{K}^{2}}\left\|p-q_{1}\right\|_{0, K}^{2}\right\}^{\frac{1}{2}}\left\|\left(\boldsymbol{e}_{\boldsymbol{u}}, e_{p}\right)\right\|_{h}+\sum_{F \in \mathcal{E}_{\Omega}}\left|\llbracket \sigma \tau_{K} \rrbracket_{F}\right|\left\|\boldsymbol{e}_{\boldsymbol{u}}\right\|_{0, F}\left\|p-q_{1}\right\|_{0, F} .
\end{aligned}
$$

Next, using the local trace result (cf. [35]): there exists $C>0$, independent of $h$, such that, for all $K \in \mathcal{T}_{h}, F \in \mathcal{E}_{K}$ and all $v \in H^{1}\left(\omega_{F}\right)$

$$
\|v\|_{0, F} \leq C\left(h_{F}^{-\frac{1}{2}}\|v\|_{0, \omega_{F}}+h_{F}^{\frac{1}{2}}|v|_{1, \omega_{F}}\right)
$$

the regularity of the mesh, (41), (42) and the definition of $\alpha_{K}$, we obtain

$$
\begin{aligned}
& \sum_{F \in \mathcal{E}_{\Omega}}\left|\llbracket \sigma \tau_{K} \rrbracket_{F}\right|\left\|\boldsymbol{e}_{\boldsymbol{u}}\right\|_{0, F}\left\|p-q_{1}\right\|_{0, F} \\
\leq & C \sum_{F \in \mathcal{E}_{\Omega}}\left|\llbracket \sigma \tau_{K} \rrbracket_{F}\right|\left\{h_{F}^{-\frac{1}{2}}\left\|\boldsymbol{e}_{\boldsymbol{u}}\right\|_{0, \omega_{F}}+h_{F}^{\frac{1}{2}}\left|\boldsymbol{e}_{\boldsymbol{u}}\right|_{1, \omega_{F}}\right\}\left\{h_{F}^{-\frac{1}{2}}\left\|p-q_{1}\right\|_{0, \omega_{F}}+h_{F}^{\frac{1}{2}}\left|p-q_{1}\right|_{1, \omega_{F}}\right\} \\
= & C \sum_{F \in \mathcal{E}_{\Omega}}\left|\llbracket 1-\sigma \tau_{K} \rrbracket_{F}\right|\left\{\left\|\boldsymbol{e}_{\boldsymbol{u}}\right\|_{0, \omega_{F}}+h_{F}\left|\boldsymbol{e}_{\boldsymbol{u}}\right|_{1, \omega_{F}}\right\}\left\{h_{F}^{-1}\left\|p-q_{1}\right\|_{0, \omega_{F}}+\left|p-q_{1}\right|_{1, \omega_{F}}\right\} \\
\leq & C\left\{\sum_{K \in \mathcal{T}_{h}}\left(1-\sigma \tau_{K}\right)\left\|\boldsymbol{e}_{\boldsymbol{u}}\right\|_{0, K}^{2}+\frac{h_{K}^{2}}{1+\alpha_{K}^{2}}\left|\boldsymbol{e}_{\boldsymbol{u}}\right|_{1, K}^{2}\right\}^{\frac{1}{2}} \times q^{\frac{1}{2}} \times q^{\frac{1}{2}}\left\|\left(\boldsymbol{e}_{\boldsymbol{u}}, e_{p}\right)\right\|_{h} . \\
& \left\{\sum_{K \in \mathcal{T}_{h}} \min \left\{1, \alpha_{K}^{2}\right\}\left(h_{K}^{-2}\left\|p-q_{1}^{2}\right\|_{0, K}^{2}+\mid p-q_{1, K}\right)\right. \\
\leq & \left\{\sum_{K \in \mathcal{T}_{h}} \frac{\min \left\{1, \alpha_{K}^{2}\right\}}{\sigma}\left(h_{K}^{-2}\left\|p-q_{1}\right\|_{0, K}^{2}+\left|p-q_{1}\right|_{1, K}^{2}\right)\right\}^{\frac{1}{2}}
\end{aligned}
$$


Finally, in an analogous way arrive at the following

$$
\begin{aligned}
& I I I=\sum_{K \in \mathcal{T}_{h}} \nu \tau_{K}\left(\Delta \boldsymbol{u}, \sigma\left(\boldsymbol{v}_{1}-\boldsymbol{u}_{1}\right)\right)_{K}=\sum_{K \in \mathcal{T}_{h}} \nu \tau_{K}\left(\Delta \boldsymbol{u}, \sigma \boldsymbol{e}_{\boldsymbol{u}}\right)_{K}+\sum_{K \in \mathcal{T}_{h}} \nu \tau_{K}\left(\Delta \boldsymbol{u}, \sigma\left(\boldsymbol{u}-\boldsymbol{v}_{1}\right)\right)_{K} \\
\leq & \left.C \sum_{K \in \mathcal{T}_{h}} \frac{\nu^{2} \tau_{K}^{2} \sigma}{1-\sigma \tau_{K}}|\boldsymbol{u}|_{2, K}^{2}\right\}^{\frac{1}{2}}\left\|\left(\boldsymbol{e}_{\boldsymbol{u}}, e_{p}\right)\right\|_{h}+C \sum_{K \in \mathcal{T}_{h}} \nu \sigma \tau_{K}|\boldsymbol{u}|_{2, K}\left\|\boldsymbol{u}-\boldsymbol{v}_{1}\right\|_{0, K} \\
\leq & C\left\{\sum_{K \in \mathcal{T}_{h}} \nu^{2} \frac{\min \left\{1, \alpha_{K}^{2}\right\}}{\sigma}\left(1+\alpha_{K}^{2}\right)|\boldsymbol{u}|_{2, K}^{2}\right\}^{\frac{1}{2}}\left\|\left(\boldsymbol{e}_{\boldsymbol{u}}, e_{p}\right)\right\|_{h}+C \sum_{K \in \mathcal{T}_{h}} \nu|\boldsymbol{u}|_{2, K}\left\|\boldsymbol{u}-\boldsymbol{v}_{1}\right\|_{0, K} \\
\leq & C\left\{\sum_{K \in \mathcal{T}_{h}} \frac{\nu^{2}}{\sigma} \min \left\{1, \alpha_{K}^{2}\right\} \max \left\{1, \alpha_{K}^{2}\right\}|\boldsymbol{u}|_{2, K}^{2}\right\}^{\frac{1}{2}}\left\|\left(\boldsymbol{e}_{\boldsymbol{u}}, e_{p}\right)\right\|_{h}+C \sum_{K \in \mathcal{T}_{h}} \nu|\boldsymbol{u}|_{2, K}\left\|\boldsymbol{u}-\boldsymbol{v}_{1}\right\|_{0, K} \\
= & C\left\{\sum_{K \in \mathcal{T}_{h}} \frac{\nu^{2} \alpha_{K}^{2}}{\sigma}|\boldsymbol{u}|_{2, K}^{2}\right\}^{\frac{1}{2}}\left\|\left(\boldsymbol{e}_{\boldsymbol{u}}, e_{p}\right)\right\|_{h}+C \sum_{K \in \mathcal{T}_{h}} \nu|\boldsymbol{u}|_{2, K}\left\|\boldsymbol{u}-\boldsymbol{v}_{1}\right\|_{0, K} .
\end{aligned}
$$

Summing up, from (44)-(48), and the definition of $\alpha_{K}$, we obtain

$$
\begin{aligned}
& \left\|\left(\boldsymbol{e}_{\boldsymbol{u}}, e_{p}\right)\right\|_{h}^{2} \leq\left\|\left(\boldsymbol{e}_{\boldsymbol{u}}, e_{p}\right)\right\|_{h}\left\|\left(\boldsymbol{u}-\boldsymbol{v}_{1}, p-q_{1}\right)\right\|_{h} \\
& +C\left\{\sum_{K \in \mathcal{T}_{h}} \sigma \alpha_{K}^{-2}\left\|\boldsymbol{u}-\boldsymbol{v}_{1}\right\|_{0, K}^{2}\right\}^{\frac{1}{2}}\left\|\left(\boldsymbol{e}_{\boldsymbol{u}}, e_{p}\right)\right\|_{h}+C\left\{\sum_{K \in \mathcal{T}_{h}} \frac{\nu^{-1}}{1+\alpha_{K}^{2}}\left\|p-q_{1}\right\|_{0, K}^{2}\right\}^{\frac{1}{2}}\left\|\left(\boldsymbol{e}_{\boldsymbol{u}}, e_{p}\right)\right\|_{h} \\
& +C\left\{\sum_{K \in \mathcal{T}_{h}} \frac{\min \left\{1, \alpha_{K}^{2}\right\}}{\sigma}\left(h_{K}^{-2}\left\|p-q_{1}\right\|_{0, K}^{2}+\left|p-q_{1}\right|_{1, K}^{2}\right)\right\}^{\frac{1}{2}}\left\|\left(\boldsymbol{e}_{\boldsymbol{u}}, e_{p}\right)\right\|_{h} \\
& +C\left\{\sum_{K \in \mathcal{T}_{h}} \frac{\nu^{2} \alpha_{K}^{2}}{\sigma}|\boldsymbol{u}|_{2, K}^{2}\right\}^{\frac{1}{2}}\left\|\left(\boldsymbol{e}_{\boldsymbol{u}}, e_{p}\right)\right\|_{h}+C \sum_{K \in \mathcal{T}_{h}} \nu|\boldsymbol{u}|_{2, K}\left\|\boldsymbol{u}-\boldsymbol{v}_{1}\right\|_{0, K} \\
& +C h \sqrt{\nu}|\boldsymbol{u}|_{2, \Omega}\left\|\left(\boldsymbol{e}_{\boldsymbol{u}}, e_{p}\right)\right\|_{h}+C h \sqrt{\nu}|\boldsymbol{u}|_{2, \Omega}\left\|\left(\boldsymbol{u}-\boldsymbol{v}_{1}, p-q_{1}\right)\right\|_{h} \\
& \leq C\left\{\| \boldsymbol{u}-\boldsymbol{v}_{1}, p-q_{1}\right) \|_{h}^{2}+\sum_{K \in \mathcal{T}_{h}}\left(\sigma \alpha_{K}^{-2}\left\|\boldsymbol{u}-\boldsymbol{v}_{1}\right\|_{0, K}^{2}+\nu|\boldsymbol{u}|_{2, K}\left\|\boldsymbol{u}-\boldsymbol{v}_{1}\right\|_{0, K}\right)+\nu h^{2}|\boldsymbol{u}|_{2, \Omega}^{2} \\
& \left.+\sum_{K \in \mathcal{T}_{h}} \frac{\nu^{-1}}{1+\alpha_{K}^{2}}\left\|p-q_{1}\right\|_{0, K}^{2}+\sum_{K \in \mathcal{T}_{h}} \frac{\min \left\{1, \alpha_{K}^{2}\right\}}{\sigma}\left(h_{K}^{-2}\left\|p-q_{1}\right\|_{0, K}^{2}+\left|p-q_{1}\right|_{1, K}^{2}\right)\right\} \\
& +\frac{1}{2}\left\|\left(\boldsymbol{e}_{\boldsymbol{u}}, e_{p}\right)\right\|_{h}^{2},
\end{aligned}
$$


and the result follows using that

$$
\frac{\nu^{-1}}{1+\alpha_{K}^{2}} \leq C \frac{\min \left\{1, \alpha_{K}^{2}\right\} h_{K}^{-2}}{\sigma},
$$

and rearranging terms.

For the proof of the next result we introduce the Clément interpolation operator (cf. $[18,19]) \mathcal{C}_{h}: H^{1}(\Omega) \rightarrow V_{h}\left(\right.$ if $v \in H_{0}^{1}(\Omega)$, then we may define $\mathcal{C}_{h}(v)$ with values in $\left.V_{h} \cap H_{0}^{1}(\Omega)\right)$, satisfying

$$
\begin{gathered}
\left\|v-\mathcal{C}_{h}(v)\right\|_{0, K} \leq C\|v\|_{0, \omega_{K}}, \\
\left|v-\mathcal{C}_{h}(v)\right|_{m, K} \leq C h_{K}^{1-m}|v|_{1, \omega_{K}},
\end{gathered}
$$

for $m=0,1$, with the obvious extension to vector-valued functions.

Lemma 5. Let us suppose that $(\boldsymbol{u}, p) \in\left[H^{2}(\Omega) \cap H_{0}^{1}(\Omega)\right]^{2} \times\left[H^{1}(\Omega) \cap L_{0}^{2}(\Omega)\right]$ is the solution of (14). Then, there exists $C>0$ such that

$$
\begin{gathered}
\left\|\left(\boldsymbol{u}-\mathcal{C}_{h}(\boldsymbol{u}), p-\tilde{p}_{h}\right)\right\|_{h}^{2}+\sum_{K \in \mathcal{T}_{h}} \nu h_{K}^{-2}\left\|\boldsymbol{u}-\mathcal{C}_{h}(\boldsymbol{u})\right\|_{0, K}^{2} \\
+\sum_{K \in \mathcal{T}_{h}} \frac{\min \left\{1, \alpha_{K}^{2}\right\}}{\sigma}\left(h_{K}^{-2}\left\|p-\tilde{p}_{h}\right\|_{0, K}^{2}+\left|p-\tilde{p}_{h}\right|_{1, K}^{2}\right) \leq C h^{2} \nu|\boldsymbol{u}|_{2, \Omega}^{2}+\frac{\min \left\{1, \alpha_{K}^{2}\right\}}{\sigma}|p|_{1, \Omega}^{2}
\end{gathered}
$$

where $\tilde{p}_{h}=\mathcal{C}_{h}(p)-\frac{\left(\mathcal{C}_{h}(p), 1\right)_{\Omega}}{|\Omega|} \in Q_{h}$.

Proof. The result follows from the definition of the norm $\|\cdot\|_{h}$. Indeed, using (49)-(50), (40)-(41) and the regularity of the mesh we obtain

$$
\begin{aligned}
\left\|\left(\boldsymbol{u}-\mathcal{C}_{h}(\boldsymbol{u}), p-\tilde{p}_{h}\right)\right\|_{h}^{2} & =\sum_{K \in \mathcal{T}_{h}} \sigma\left(1-\sigma \tau_{K}\right)\left\|\boldsymbol{u}-\mathcal{C}_{h}(\boldsymbol{u})\right\|_{0, K}^{2}+\nu\left|\boldsymbol{u}-\mathcal{C}_{h}(\boldsymbol{u})\right|_{1, K}^{2}+\tau_{K}\left|p-\tilde{p}_{h}\right|_{1, K}^{2} \\
& \leq C \sum_{K \in \mathcal{T}_{h}}\left[\left(\frac{\sigma h_{K}^{4}}{1+\alpha_{K}^{2}}+\nu h_{K}^{2}\right)|\boldsymbol{u}|_{2, \omega_{K}}^{2}+\frac{\min \left\{1, \alpha_{K}^{2}\right\}}{\sigma}|p|_{1, \omega_{K}}^{2}\right] \\
& \leq C\left(\nu h^{2}|\boldsymbol{u}|_{2, \Omega}^{2}+\frac{\min \left\{1, \alpha_{K}^{2}\right\}}{\sigma}|p|_{1, \Omega}^{2}\right) .
\end{aligned}
$$

The other terms are bounded in a similar way.

Finally, using the previous result and the asymptotic behavior of $\tau_{K}$ (cf. Lemma 1) we can prove the following optimal convergence result. 
Theorem 6. Let us suppose that $(\boldsymbol{u}, p) \in\left[H^{2}(\Omega) \cap H_{0}^{1}(\Omega)\right]^{2} \times\left[H^{1}(\Omega) \cap L_{0}^{2}(\Omega)\right]$ is the solution of (14) and that $\left(\boldsymbol{u}_{1}, p_{1}\right) \in \mathbf{V}_{h} \times Q_{h}$ is the solution of (35). Then, there exists $C>0$ independent of $h, \sigma$ and $\nu$, such that

$$
\left\|\left(\boldsymbol{e}_{\boldsymbol{u}}, e_{p}\right)\right\|_{h} \leq C\left(\sqrt{\nu} h|\boldsymbol{u}|_{2, \Omega}+\frac{\min \left\{1, \alpha_{K}\right\}}{\sqrt{\sigma}}|p|_{1, \Omega}\right)
$$

Proof. The result follows applying Lemmas 4 and 5 with $\boldsymbol{v}_{1}=\mathcal{C}_{h}(\boldsymbol{u})$ and $q_{1}=\tilde{p}_{h}$.

Remark. The estimate from Theorem 6 may written as:

$$
\left[\sum_{K \in \mathcal{T}_{h}} \frac{\sigma\left(1-\sigma \tau_{K}\right)}{\nu}\left\|\boldsymbol{e}_{\boldsymbol{u}}\right\|_{0, K}^{2}+\frac{\tau_{K}}{\nu}\left|e_{p}\right|_{1, K}^{2}\right]^{\frac{1}{2}}+\left|\boldsymbol{e}_{\boldsymbol{u}}\right|_{1, \Omega} \leq C\left(h|\boldsymbol{u}|_{2, \Omega}+\frac{\min \left\{1, \alpha_{K}\right\}}{\sqrt{\sigma \nu}}|p|_{1, \Omega}\right)
$$

which, using (41) leads to

$$
\left[\sum_{K \in \mathcal{T}_{h}} \frac{\sigma}{\sigma h_{K}^{2}+\nu}\left\|\boldsymbol{e}_{\boldsymbol{u}}\right\|_{0, K}^{2}\right]^{\frac{1}{2}}+\left|\boldsymbol{e}_{\boldsymbol{u}}\right|_{1, \Omega} \leq C\left(h|\boldsymbol{u}|_{2, \Omega}+\frac{\min \left\{1, \alpha_{K}\right\}}{\sqrt{\sigma \nu}}|p|_{1, \Omega}\right)
$$

which may be seen as a robust estimate for the velocity. Now, if $\nu \leq \sigma h_{K}^{2}$, then (51) provides the following estimate

$$
\left\|\boldsymbol{e}_{\boldsymbol{u}}\right\|_{0, \Omega} \leq C h^{2}\left(|\boldsymbol{u}|_{2, \Omega}+\frac{1}{\nu}|p|_{1, \Omega}\right)
$$

which is an optimal error estimate for $\left\|\boldsymbol{e}_{\boldsymbol{u}}\right\|_{0, \Omega}$, which does not need the use of a duality argument.

In the next result we state an error estimate for the pressure in its natural norm.

Theorem 7. Under the same assumptions as in Theorem 6, there exists a positive constant $C>0$ such that

$$
\left\|e_{p}\right\|_{0, \Omega} \leq C \sqrt{\sigma} \max \{1, \sqrt{\nu}\}\left(\sqrt{\nu} h|\boldsymbol{u}|_{2, \Omega}+\frac{\min \left\{1, \alpha_{K}\right\}}{\sqrt{\sigma}}|p|_{1, \Omega}+\min \left\{1, \alpha_{K}\right\}|\boldsymbol{u}|_{2, \Omega}\right)
$$

Proof. From the continuous inf-sup condition (see [27]), there exists $\boldsymbol{w} \in H_{0}^{1}(\Omega)^{2}$ such that $\nabla \cdot \boldsymbol{w}=e_{p}$ in $\Omega$ and $\|\boldsymbol{w}\|_{1, \Omega} \leq C\left\|e_{p}\right\|_{0, \Omega}$. Let $\boldsymbol{w}_{h}=\mathcal{C}_{h}(\boldsymbol{w}) \in \mathbf{V}_{h}$ be the Clément interpolant of $\boldsymbol{w}$. Then, integrating by parts, (35) (applied to $\left(\boldsymbol{w}_{h}, 0\right)$ ) and using Cauchy-Schwarz 
inequality, we obtain

$$
\begin{aligned}
& \left\|e_{p}\right\|_{0, \Omega}^{2}=\left(\nabla \cdot \boldsymbol{w}, e_{p}\right)_{\Omega} \\
& =\left(\nabla \cdot\left(\boldsymbol{w}-\boldsymbol{w}_{h}\right), e_{p}\right)_{\Omega}+\left(\nabla \cdot \boldsymbol{w}_{h}, e_{p}\right)_{\Omega} \\
& =-\left(\boldsymbol{w}-\boldsymbol{w}_{h}, \nabla e_{p}\right)_{\Omega}+\nu\left(\nabla \boldsymbol{e}_{\boldsymbol{u}}, \nabla \boldsymbol{w}_{h}\right)_{\Omega}+\sigma\left(\boldsymbol{e}_{\boldsymbol{u}}, \boldsymbol{w}_{h}\right)_{\Omega} \\
& -\sum_{K \in \mathcal{T}_{h}} \tau_{K}\left(\sigma \boldsymbol{e}_{\boldsymbol{u}}+\nabla e_{p}, \sigma \boldsymbol{w}_{h}\right)_{K}-\sum_{K \in \mathcal{T}_{h}} \tau_{K}\left(\nu \Delta \boldsymbol{u}, \sigma \boldsymbol{w}_{h}\right)_{K} \\
& =-\left(\boldsymbol{w}-\boldsymbol{w}_{h}, \nabla e_{p}\right)_{\Omega}+\nu\left(\nabla \boldsymbol{e}_{\boldsymbol{u}}, \nabla \boldsymbol{w}_{h}\right)_{\Omega}+\sum_{K \in \mathcal{T}_{h}}\left(\left(1-\sigma \tau_{K}\right) \boldsymbol{e}_{\boldsymbol{u}}, \sigma \boldsymbol{w}_{h}\right)_{K} \\
& -\sum_{K \in \mathcal{T}_{h}} \tau_{K}\left(\nabla e_{p}, \sigma \boldsymbol{w}_{h}\right)_{K}-\sum_{K \in \mathcal{T}_{h}} \tau_{K}\left(\nu \Delta \boldsymbol{u}, \sigma \boldsymbol{w}_{h}\right)_{K} \\
& \leq C \sum_{K \in \mathcal{T}_{h}} h_{K}|\boldsymbol{w}|_{1, \omega_{K}}\left\|\nabla e_{p}\right\|_{0, K}+\nu\left|\boldsymbol{e}_{\boldsymbol{u}}\right|_{1, \Omega}\left|\boldsymbol{w}_{h}\right|_{1, \Omega}+\sum_{K \in \mathcal{T}_{h}} \sigma\left(1-\sigma \tau_{K}\right)\left\|\boldsymbol{e}_{\boldsymbol{u}}\right\|_{0, K}\left\|\boldsymbol{w}_{h}\right\|_{0, K} \\
& +\sum_{K \in \mathcal{T}_{h}} \sigma \tau_{K}\left\|\nabla e_{p}\right\|_{0, K}\left\|\boldsymbol{w}_{h}\right\|_{0, K}+\sum_{K \in \mathcal{T}_{h}} \sigma \tau_{K} \nu|\boldsymbol{u}|_{2, K}\left\|\boldsymbol{w}_{h}\right\|_{0, K} \\
& \leq C\left[\sum_{K \in \mathcal{T}_{h}}\left(\tau_{K}+\frac{\sigma^{2} \tau_{K}^{2}}{\nu}\right)\left|e_{p}\right|_{1, K}^{2}+\nu\left|\boldsymbol{e}_{\boldsymbol{u}}\right|_{1, K}^{2}+\frac{\sigma^{2}\left(1-\sigma \tau_{K}\right)^{2}}{\nu}\left\|\boldsymbol{e}_{\boldsymbol{u}}\right\|_{0, K}^{2}+\sigma^{2} \tau_{K}^{2} \nu|\boldsymbol{u}|_{2, K}^{2}\right]^{\frac{1}{2}} \times \\
& {\left[\sum_{K \in \mathcal{T}_{h}} \nu|\boldsymbol{w}|_{1, \omega_{K}}^{2}+\nu\left|\boldsymbol{w}_{h}\right|_{1, \Omega}^{2}+\nu\left\|\boldsymbol{w}_{h}\right\|_{0, \Omega}^{2}\right]^{\frac{1}{2}}} \\
& \leq C \sqrt{\sigma} \max \{1, \sqrt{\nu}\}\left[\left\|\left(\boldsymbol{e}_{\boldsymbol{u}}, e_{p}\right)\right\|_{h}^{2}+\sum_{K \in \mathcal{T}_{h}} \min \left\{1, \alpha_{K}^{4}\right\}|\boldsymbol{u}|_{2, K}^{2}\right]^{\frac{1}{2}}\left[|\boldsymbol{w}|_{1, \Omega}^{2}+\left|\boldsymbol{w}_{h}\right|_{1, \Omega}^{2}+\left\|\boldsymbol{w}_{h}\right\|_{0, \Omega}^{2}\right]^{\frac{1}{2}} .
\end{aligned}
$$

Now, using the approximation properties of the Clément interpolant (cf. [19]) we obtain

$$
\left[|\boldsymbol{w}|_{1, \Omega}^{2}+\left|\boldsymbol{w}_{h}\right|_{1, \Omega}^{2}+\left\|\boldsymbol{w}_{h}\right\|_{0, \Omega}^{2}\right]^{\frac{1}{2}} \leq C\|\boldsymbol{w}\|_{1, \Omega} \leq C\left\|e_{p}\right\|_{0, \Omega} .
$$

Hence, dividing in (53) by $\left\|e_{p}\right\|_{0, \Omega}$, and using the definition of the norm $\|\cdot\|_{h}$, we have

$$
\left\|p-p_{1}\right\|_{0, \Omega} \leq C \sqrt{\sigma} \max \{1, \sqrt{\nu}\}\left(\left\|\left(\boldsymbol{u}-\boldsymbol{u}_{1}, p-p_{1}\right)\right\|_{h}+\min \left\{1, \alpha_{K}\right\}|\boldsymbol{u}|_{2, \Omega}\right),
$$

and the result follows applying Theorem 6 .

Throughout the next lemma we will suppose that the solution of the problem: Find $(\boldsymbol{\varphi}, \pi)$ such that:

$$
\begin{aligned}
\sigma \boldsymbol{\varphi}-\nu \Delta \boldsymbol{\varphi}-\nabla \pi=\boldsymbol{u}-\boldsymbol{u}_{1} & , \quad \nabla \cdot \boldsymbol{\varphi}=0 \quad \text { in } \Omega \\
\boldsymbol{\varphi} & =\mathbf{0} \quad \text { on } \partial \Omega,
\end{aligned}
$$


where $\left(\boldsymbol{u}_{1}, p_{1}\right)$ is the solution of $(35)$, belongs to $\left[H^{2}(\Omega) \cap H_{0}^{1}(\Omega)\right]^{2} \times\left[H^{1}(\Omega) \cap L_{0}^{2}(\Omega)\right]$, and that there exists a constant $C$, possibly depending on $\sigma$ and $\nu$, but not on $h$, such that

$$
\|\boldsymbol{\varphi}\|_{2, \Omega}+\|\pi\|_{1, \Omega} \leq C\left\|\boldsymbol{u}-\boldsymbol{u}_{1}\right\|_{0, \Omega}
$$

Theorem 8. Under the same assumptions of Theorem 6 the following error estimates hold: If $\nu \leq \sigma h_{K}^{2}$, then there exists $C>0$, independent of $h, \sigma$ and $\nu$, such that

$$
\left\|\boldsymbol{e}_{\boldsymbol{u}}\right\|_{0, \Omega} \leq C h^{2}\left(|\boldsymbol{u}|_{2, \Omega}+\frac{1}{\nu}|p|_{1, \Omega}\right)
$$

If $\sigma h_{K}^{2}<\nu$, then there exists $C>0$, independent of $h$, but depending on $\sigma$ and $\nu$, such that

$$
\left\|\boldsymbol{e}_{\boldsymbol{u}}\right\|_{0, \Omega} \leq C h^{2}\left(|\boldsymbol{u}|_{2, \Omega}+|p|_{1, \Omega}\right)
$$

Proof. Since we only need to prove the diffusive-dominated case $\left(\sigma h_{K}^{2}<\nu\right)$, then we will treat $\sigma$ and $\nu$ as fixed constants. Let $\left(\boldsymbol{\varphi}_{h}, \pi_{h}\right):=\left(\mathcal{C}_{h}(\boldsymbol{\varphi}), \mathcal{C}_{h}(\pi)-\frac{\left(\mathcal{C}_{h}(\pi), 1\right)_{\Omega}}{|\Omega|}\right) \in \mathbf{V}_{h} \times Q_{h}$. Then, multiplying the first equation in (54) by $\boldsymbol{u}-\boldsymbol{u}_{1}$ and second by $-\left(p-p_{1}\right)$, from the definition of the bilinear form $\mathbf{B}_{\tau}$, interpolation inequalities (50)-(49), and Theorems 6 and 7 , we obtain

$$
\begin{aligned}
\left\|\boldsymbol{u}-\boldsymbol{u}_{1}\right\|_{0, \Omega}^{2}= & \sigma\left(\boldsymbol{\varphi}, \boldsymbol{u}-\boldsymbol{u}_{1}\right)_{\Omega}+\nu\left(\nabla \boldsymbol{\varphi}, \nabla\left(\boldsymbol{u}-\boldsymbol{u}_{1}\right)\right)_{\Omega}+\left(\pi, \nabla \cdot\left(\boldsymbol{u}-\boldsymbol{u}_{1}\right)\right)_{\Omega}-\left(p-p_{1}, \nabla \cdot \boldsymbol{\varphi}\right)_{\Omega} \\
= & \mathbf{B}_{\tau}\left(\left(\boldsymbol{u}-\boldsymbol{u}_{1}, p-p_{1}\right),(\boldsymbol{\varphi}, \pi)\right)+\sum_{K \in \mathcal{T}_{h}} \tau_{K}\left(\sigma\left(\boldsymbol{u}-\boldsymbol{u}_{1}\right)+\nabla\left(p-p_{1}\right), \sigma \boldsymbol{\varphi}+\nabla \pi\right)_{K} \\
= & \mathbf{B}_{\tau}\left(\left(\boldsymbol{u}-\boldsymbol{u}_{1}, p-p_{1}\right),\left(\boldsymbol{\varphi}-\boldsymbol{\varphi}_{h}, \pi-\pi_{h}\right)\right)+\sum_{K \in \mathcal{T}_{h}} \tau_{K} \nu\left(\Delta \boldsymbol{u}, \sigma \boldsymbol{\varphi}_{h}-\nabla \pi_{h}\right)_{K} \\
& +\sum_{K \in \mathcal{T}_{h}} \tau_{K}\left(\sigma\left(\boldsymbol{u}-\boldsymbol{u}_{1}\right)+\nabla\left(p-p_{1}\right), \nu \Delta \boldsymbol{\varphi}+\left(\boldsymbol{u}-\boldsymbol{u}_{1}\right)\right)_{K} \\
\leq & \left.C\left\|\left(\boldsymbol{u}-\boldsymbol{u}_{1}, p-p_{1}\right)\right\|_{h}^{2}+\left\|\boldsymbol{u}-\boldsymbol{u}_{1}\right\|_{0, \Omega}^{2}+h^{2}|\boldsymbol{u}|_{2, \Omega}^{2}+\left\|p-p_{1}\right\|_{0, \Omega}^{2}\right\}^{\frac{1}{2}} \times \\
& \left\{\sum_{K \in \mathcal{T}_{h}}\left\|\boldsymbol{\varphi}-\boldsymbol{\varphi}_{h}\right\|_{0, K}^{2}+\left|\boldsymbol{\varphi}-\boldsymbol{\varphi}_{h}\right|_{1, K}^{2}+\left\|\pi-\pi_{h}\right\|_{0, K}^{2}+\tau_{K}\left\|\nabla\left(\pi-\pi_{h}\right)\right\|_{0, K}^{2}\right. \\
& \left.+h^{2}\left\|\varphi_{h}\right\|_{0, K}^{2}+h^{2}\left|\pi_{h}\right|_{1, K}^{2}+\tau_{K}|\boldsymbol{\varphi}|_{2, K}^{2}+\tau_{K}\left\|\boldsymbol{u}-\boldsymbol{u}_{1}\right\|_{0, K}^{2}\right\}^{\frac{1}{2}} \\
\leq & C h^{2}\left(|\boldsymbol{u}|_{2, \Omega}+\frac{1}{\sqrt{\nu}}|p|_{1, \Omega}\right)\left(|\boldsymbol{\varphi}|_{2, \Omega}^{2}+|\pi|_{1, \Omega}^{2}+\left\|\boldsymbol{u}-\boldsymbol{u}_{1}\right\|_{0, \Omega}^{2}\right)^{\frac{1}{2}},
\end{aligned}
$$

and the result follows applying (55) and dividing by $\left\|\boldsymbol{u}-\boldsymbol{u}_{1}\right\|_{0, \Omega}$. 


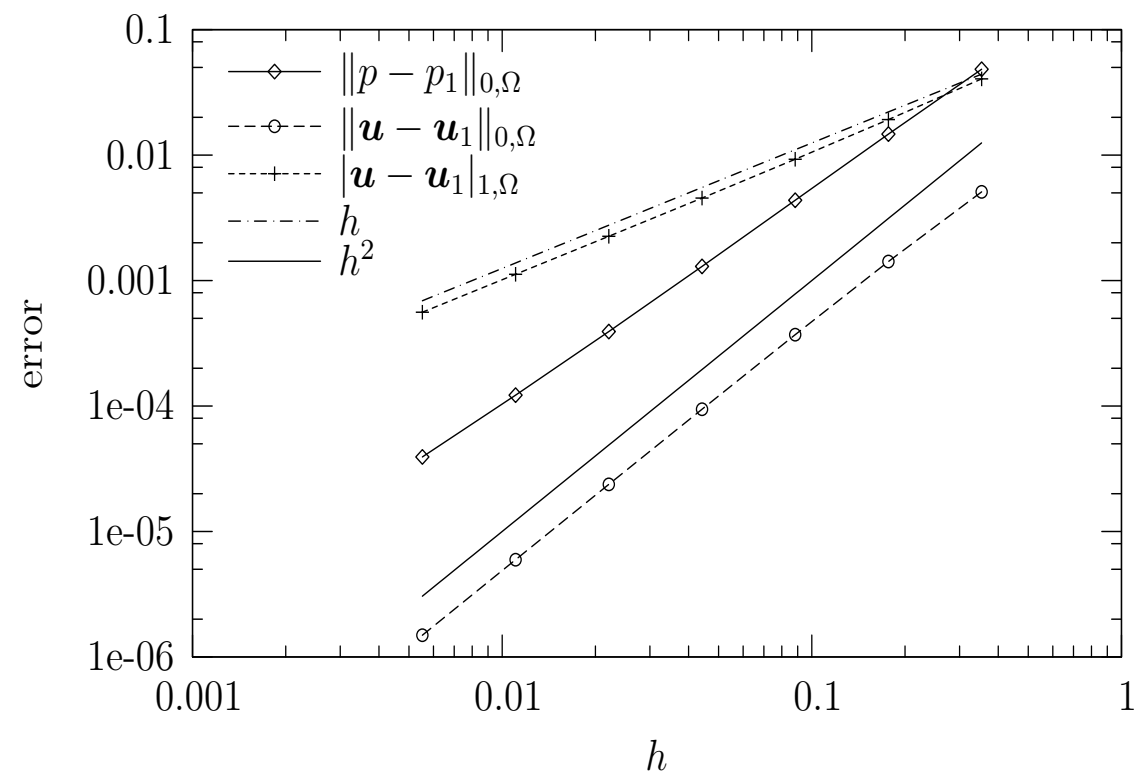

Figure 2. Convergence history for $\sigma=1$ and $\nu=1$.

\section{NumERICAL VALIDATIONS}

4.1. A problem with an analytical solution. We first perform a convergence validation. To do this, we set $\Omega=(0,1) \times(0,1)$ and $\boldsymbol{f}$ and the boundary conditions such that the exact solution of (14) is given by

$$
\begin{aligned}
\boldsymbol{u}(x, y) & =\left(\frac{\sinh \left(\sqrt{\frac{\sigma}{\nu}} y\right)}{\sinh \left(\sqrt{\frac{\sigma}{\nu}}\right)}, 0\right)^{t}, \\
p(x, y) & =(x-0.5)(y-0.5)
\end{aligned}
$$

In Figures 2-4 we depict the convergence history as $h \rightarrow 0$ for all the variables for $\sigma=1$ and $\nu=1,10^{-2}$ and $10^{-4}$, respectively, where we see that all the variables converge as predicted by the theory.

4.2. The lid-driven cavity flow. Next, we address the lid-driven cavity problem, with domain $\Omega$ as before, $\boldsymbol{f}=\mathbf{0}$, and, in order to test the performance of the method for the large $\sigma$ case, we perform experiments with $\sigma=1$ and $\sigma=10^{4}$, both using $\nu=1$. We depict in Figure 5 elevations for the pressure field and in Figure 6 of the horizontal velocity, for an unstructured (and very close to equilateral) mesh. We observe the absence of oscillations for 


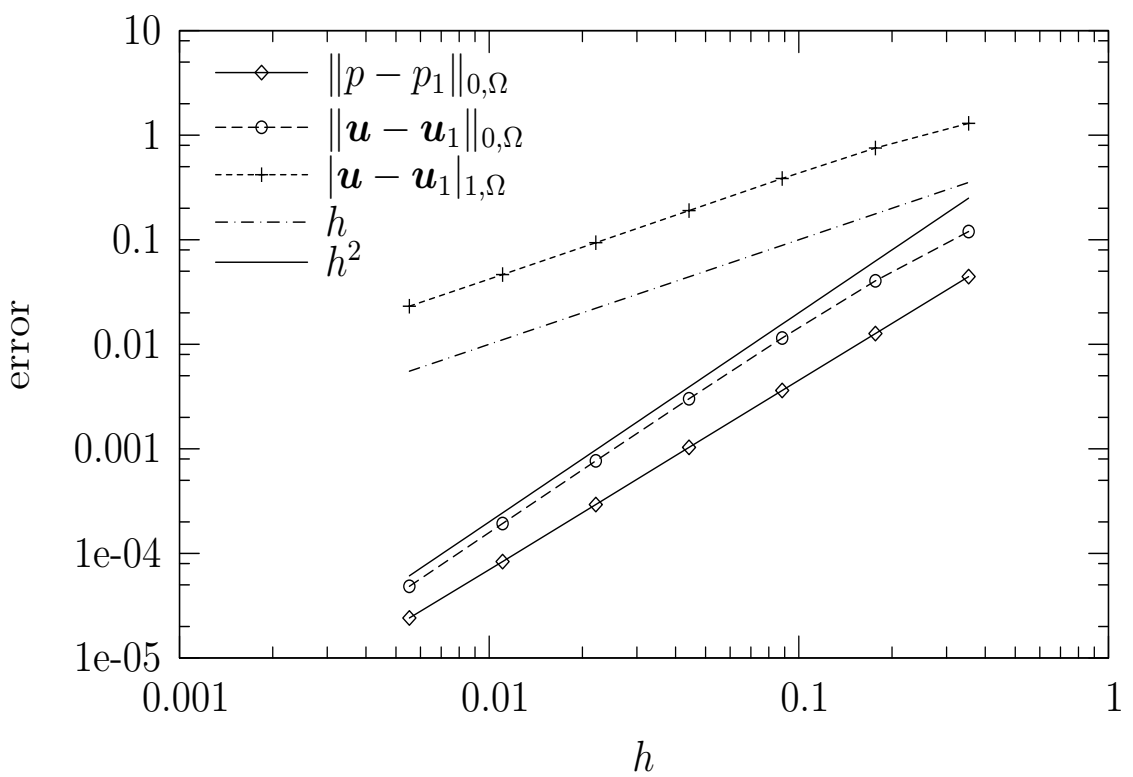

Figure 3. Convergence history for $\sigma=1$ and $\nu=10^{-2}$.

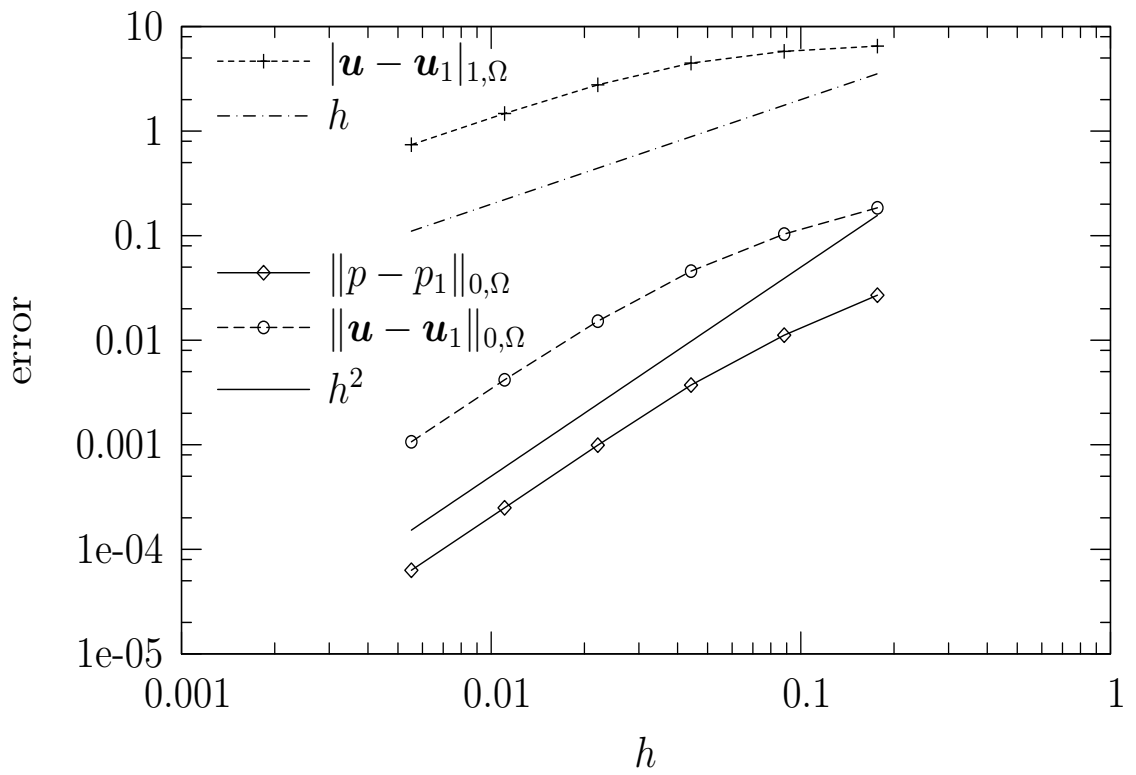

FiguRE 4. Convergence history for $\sigma=1$ and $\nu=10^{-4}$.

the pressure in both cases, which shows that the method treats well the inf-sup condition and the presence of a boundary layer for the reaction-dominated regime. 
PRESSURE

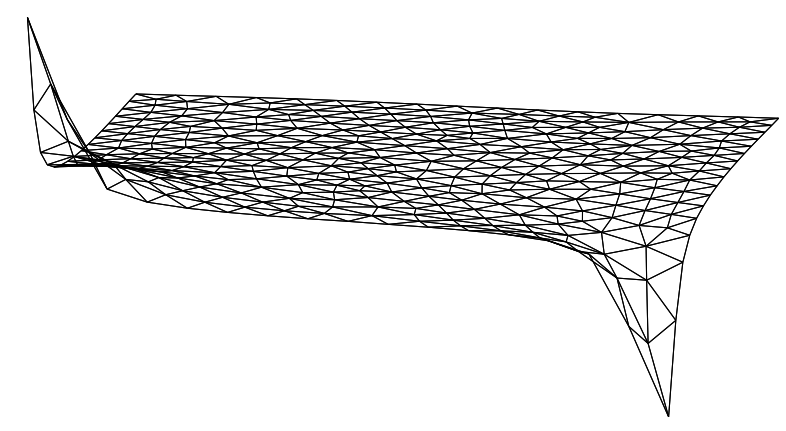

PRESSURE

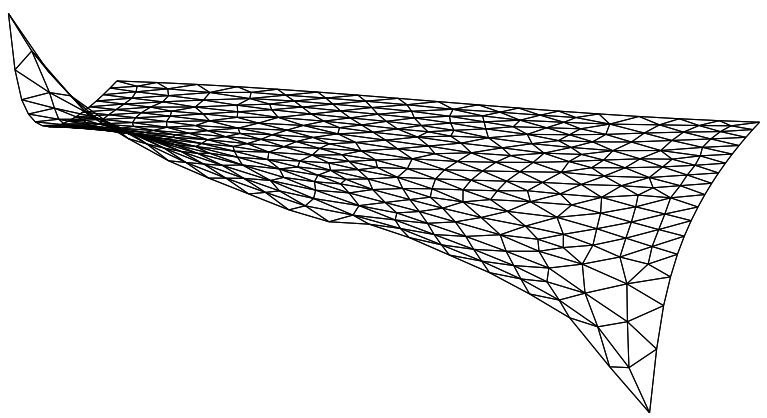

Figure 5. Pressure elevation for $\nu=1$ and $\sigma=1$ (left) and $\sigma=10^{4}$ (right).

Next, we consider a structured mesh and test the method for $\sigma=10^{4}, \nu=1$. We see in Figure 7 that a small oscillation appears. This unexpected fact deserves further investigation, but, we also remark that this oscillation may be corrected by changing the definition of $\alpha_{i}$ as follows:

$$
\alpha_{i}:=\sqrt{\frac{8 \sigma|K|^{2}}{\nu\left|F_{i}\right|^{2}}} .
$$

This fact may be explained as follows, although the method was justified for a regular mesh, we recall that the derivation was performed supposing an equilateral mesh, and the regular mesh we used for this example differs from the equilateral case.

Acknowledgments. A part of this work was done during the stay of F. Valentin at the Departamento de Ingeniería Matemática of Universidad de Concepción, Chile, and the stay of G. Barrenechea at LNCC, Petrópolis, Brazil, in the framework of the joint Chile(CONICYT)-Brazil(CNPq) project No. 2005-073 (Chile)-490639/2005-4 (Brazil). Also, the authors want to thank the anonymous referees for their valuable comments.

\section{REFERENCES}

[1] R. Araya, G. R. Barrenechea, and F. Valentin, Stabilized finite element methods based on multiscale enrichement for the Stokes problem, SIAM J. Numer. Anal., 44 (2006), pp. 322-348. 
HORIZONTAL VELOCITY

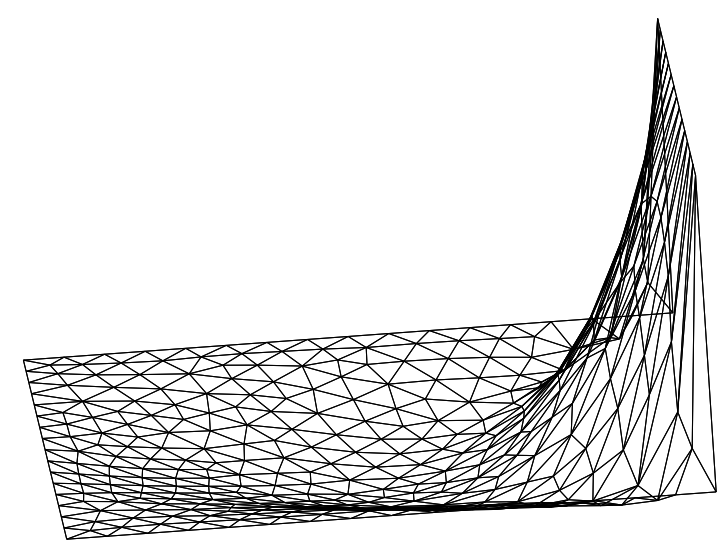

HORIZONTAL VELOCITY

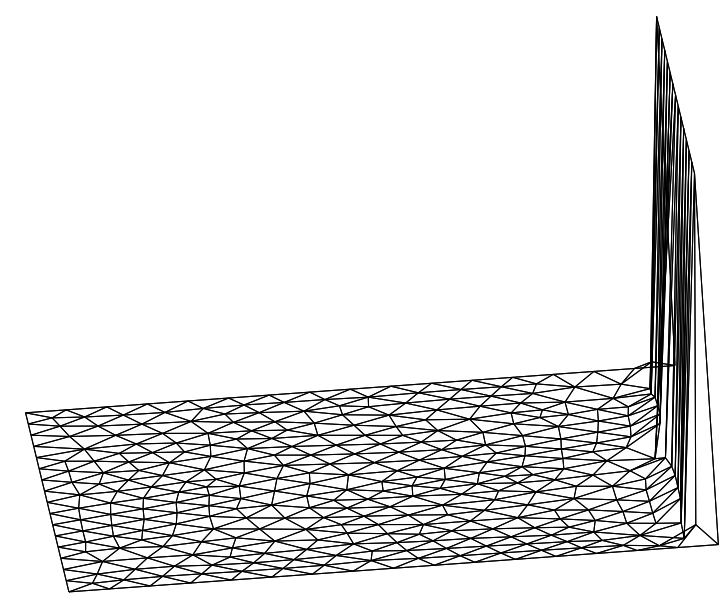

Figure 6. Elevation of the horizontal velocity for $\nu=1$ and $\sigma=1$ (left) and $\sigma=10^{4}$ (right).

\section{HORIZONTAL VELOCITY}

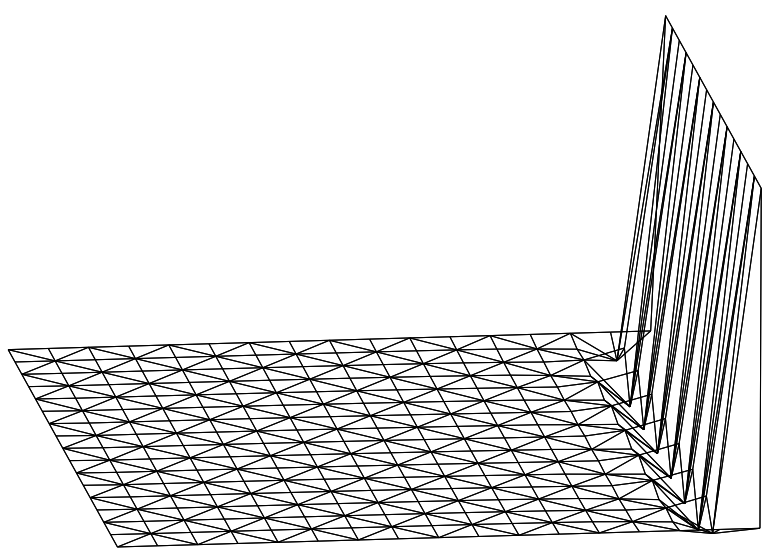

\section{HORIZONTAL VELOCITY}

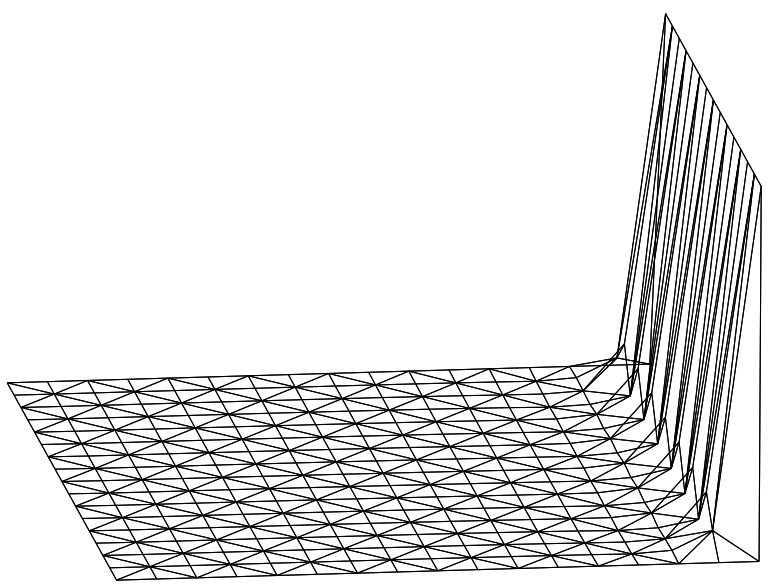

FiguRE 7. Elevation of the horizontal velocity for the standard definition of $\alpha_{i}$ (left) and (56) (right).

[2] —_, A stabilized finite element method for the Stokes problem including element and edge residuals, IMA Journal of Numerical Analysis, 27 (2007), pp. 172-197. 
[3] R. Araya and F. Valentin, A multiscale a-posteriori error estimator, Comput. Methods Appl. Mech. Engrg., 194 (2005), pp. 2077-2094.

[4] D. Arnold, F. Brezzi, And M. Fortin, A stable finite element for the Stokes equations, Calcolo, 21 (1984), pp. 337-344.

[5] C. Baiocchi, F. Brezzi, and L. Franca, Virtual bubbles and Galerkin-least-squares type methods (Ga. L. S.), Comput. Methods Appl. Mech. Engrg., 105 (1993), pp. 125-141.

[6] R. Bank and D. Welfert, A comparison between the mini-element and the Petrov-Galerkin formulations for the generalized Stokes problem, Comput. Methods Appl. Mech. Engrg., 83 (1990), pp. 61-68.

[7] G. R. Barrenechea, L. P. Franca, and F. Valentin, A Petrov-Galerkin enriched method: A mass conservative finite element method for the Darcy equation, Comput. Methods Appl. Mech. Engrg., 21-24 (2007), pp. 2449-2464.

[8] G. R. Barrenechea and F. Valentin, An unusual stabilized finite element method for a generalized Stokes problem, Numer. Math., 92 (2002), pp. 653-677.

[9] —_ Relationship between multiscale enrichment and stabilized finite element methods for the generalized Stokes problem, C. R. Math. Acad. Sci. Paris, 341 (2005), pp. 635-640.

[10] F. BrezzI, On the existence, uniqueness and approximation of saddle-point problems arising from Lagrange multipliers, RAIRO Anal. Numer., 8 (1974), pp. 129-151.

[11] F. Brezzi, M. O. Bristeau, L. P. Franca, M. Mallet, and G. Rogé, A relationship between stabilized finite element methods and the Galerkin method with bubble functions, Comput. Methods Appl. Mech. Engrg., 96 (1992), pp. 117-129.

[12] F. Brezzi And M. Fortin, Mixed and Hybrid Finite Element Methods, Springer-Verlag, 1991.

[13] F. Brezzi, L. P. Franca, T. J. R. Hughes, and A. Russo, $b=\int g$, Comput. Methods Appl. Mech. Engrg., 145 (1997), pp. 329-339.

[14] F. Brezzi, L. P. Franca, And A. Russo, Further considerations on residual-free bubbles for advectivediffusive equations, Comput. Methods Appl. Mech. Engrg., 166 (1998), pp. 25-33.

[15] F. Brezzi And A. Russo, Choosing bubbles for advection-diffusion problems, Math. Models Methods Appl. Sci., 4 (1994), pp. 571-587.

[16] A. N. Brooks and T. J. R. Hughes, Streamline upwind Petrov-Galerkin formulations for convective dominated flows with particular emphasis on the incompressible Navier-Stokes equations, Comput. Methods Appl. Mech. Engrg., 32 (1982), pp. 199-259.

[17] A. Cangiani and E. Süli, Enhanced RFB method, Numer. Math., 101 (2005), pp. 273-308.

[18] P. Clément, Approximation by finite element functions using local regularization, R.A.I.R.O. Anal. Numer., 9 (1975), pp. 77-84.

[19] A. Ern and J. L. Guermond, Theory and Practice of Finite Elements, Springer-Verlag, 2004.

[20] L. P. Franca, S. L. Frey, and T. J. R. Hughes, Stabilized finite element methods: I. Application to the advective-diffusive model, Comput. Methods Appl. Mech. Engrg., 95 (1992), pp. 253-276.

[21] L. P. Franca, A. L. Madureira, L. Tobiska, and F. Valentin, Convergence analysis of a multiscale finite element method for singularly perturbed problems, SIAM Multiscale Model. Simul., 4 (2005), pp. 839-866. 
[22] L. P. Franca, A. L. Madureira, and F. Valentin, Towards multiscale functions: enriching finite element spaces with local but not bubble-like functions, Comput. Methods Appl. Mech. Engrg., 194 (2005), pp. 3006-3021.

[23] L. P. Franca, J. V. A. Ramalho, and F. Valentin, Multiscale and residual-free bubble functions for reaction-advection-diffusion problems, International Journal for Multiscale Enginnering, 3 (2005), pp. 297-312.

[24] L. P. Franca, J. V. A. Ramalho, and F. Valentin, Multiscale finite element methods for unsteady reaction-diffusion problems, Communications in Numerical Methods in Engineering, 22 (2006), pp. 619625.

[25] L. P. Franca And A. Russo, Approximation of the Stokes problem by residual-free macro bubbles, East-West J. Numer. Math., 4 (1996), pp. 265-278.

[26] L. P. Franca and R. Stenberg, Error analysis of some Galerkin least squares methods for the elasticity equations, SIAM J. Numer. Anal., 28 (1991), pp. 1680-1697.

[27] V. Girault And P. A. Raviart, Finite Element Methods for the Navier-Stokes Equations, SpringerVerlag, 1986.

[28] T. J. R. Hughes And L. P. FrancA, A new finite element formulation for computational fluid dynamics: VII. The Stokes problem with various well-posed boundary conditions: Symmetric formulations that converge for all velocity/pressure spaces, Comput. Methods Appl. Mech. Engrg., 65 (1987), pp. 85-96.

[29] T. J. R. Hughes, L. P. Franca, and M. Balestra, A new finite element formulation for computational fluids dynamics: V. Circumventing the Babuska-Brezzi condition : A stable Petrov-Galerkin formulation of the Stokes problem accommodating equal-order interpolations, Comput. Methods Appl. Mech. Engrg., 59 (1986), pp. 85-99.

[30] T. Linb And M. Stynes, The SDFEM on Shishkin meshes for linear convection-diffusion problems, Numerich Mathematik, 87 (2001), pp. 457-484.

[31] R. Pierre, Simple $C^{0}$ approximations for the computation of incompressible flows, Comput. Methods Appl. Mech. Engrg., 68 (1988), pp. 205-227.

[32] J. V. A. Ramalho And F. Valentin, Stabilizing parabolic problems with time-dependent multiscale functions, Tech. Rep. 10/06, LNCC, 2006.

[33] H. Roos, M. Stynes, and L. Tobiska, Numerical Methods for Singularly Perturbed Differential Equations. Convection-Diffusion and Flow Problems, Springer-Verlag, 1996.

[34] M. Stynes, A uniformly convergent Galerkin method on a Shishkin mesh for a convection diffusion problem, Journal of Mathematical Analysis and Applications, (1997), pp. 36-54.

[35] V. Thомée, Galerkin Finite Element Methods for Parabolic Problems, Springer Verlag, 1997. 
Departamento de Ingeniería Matemática, Universidad de Concepción, Casilla 160-C, ConCEPCión, CHILE

E-mail address: raraya@ing-mat.udec.cl

Departamento de Ingeniería Matemática, Universidad de Concepción, Casilla 160-C, ConCEPCiÓn, ChILE

E-mail address: gbarrene@ing-mat.udec.cl

Department of Mathematics, University of Colorado at Denver, P.O. Box 173364, Campus Box 170 Denver, Colorado 80217-3364, USA

E-mail address: leo.franca@cudenver.edu

Departamento de Matemática Aplicada, laboratório Nacional de Computação Científica, Av. Getúlio Vargas, 333, 25651-070 Petrópolis - RJ, Brazil

E-mail address: valentin@lncc.br 